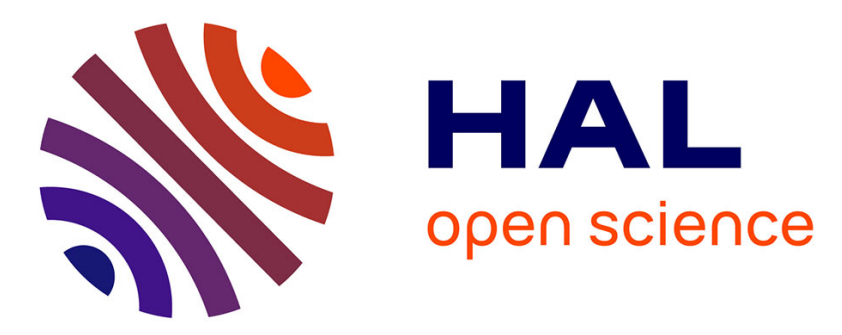

\title{
Extensive bounds on the topological entropy of repellers in piecewise expanding coupled map lattices
}

Ricardo Coutinho, Bastien Fernandez

\section{To cite this version:}

Ricardo Coutinho, Bastien Fernandez. Extensive bounds on the topological entropy of repellers in piecewise expanding coupled map lattices. Ergodic Theory and Dynamical Systems, 2013, 33 (3), pp.870-895. 10.1017/S0143385712000144 . hal-00612623

\section{HAL Id: hal-00612623 \\ https://hal.science/hal-00612623}

Submitted on 29 Jul 2011

HAL is a multi-disciplinary open access archive for the deposit and dissemination of scientific research documents, whether they are published or not. The documents may come from teaching and research institutions in France or abroad, or from public or private research centers.
L'archive ouverte pluridisciplinaire HAL, est destinée au dépôt et à la diffusion de documents scientifiques de niveau recherche, publiés ou non, émanant des établissements d'enseignement et de recherche français ou étrangers, des laboratoires publics ou privés. 


\title{
Extensive Bounds on the Entropy of Repe- llers in Expanding Coupled Map Lattices
}

\author{
Ricardo Coutinho and Bastien Fernandez
}

\begin{abstract}
Beyond the uncoupled regime, the rigorous description of the dynamics of (piecewise) expanding coupled map lattices remains largely incomplete. To address this issue, we study repellers of periodic chains of linearly coupled Lorenz-type maps which we analyze by means of symbolic dynamics. Whereas all symbolic codes are admissible for sufficiently small coupling intensity, when the interaction strength exceeds a chain length independent threshold, we prove that a large bunch of codes is pruned and an extensive decay follows suit for the topological entropy. This quantity however does not immediately drops off to 0 . Instead, it is shown to be continuous at the threshold and to remain extensively bounded below by a positive number in a large part of the expanding regime. The analysis is firstly accomplished in a piecewise affine setting where all calculations are explicit and is then extended by continuation to CML based on $C^{1}$-perturbations of the individual map.
\end{abstract}

Mathematics Subject Classification (2010). Primary 37L60; Secondary 37D50,37B40.

Keywords. Coupled Map Lattices, Piecewise Expanding Dynamical Systems, Topological Entropy.

\section{Introduction}

Coupled Map Lattices (CML) were introduced almost thirty years ago to simulate the time evolution of spatially extended systems [15]. Often designated as "discrete time dynamical systems with discrete space", their dynamics is generated by the iterations of a mapping acting on real sequences. In applications, these sequences usually represent the spatial profile of a physical quantity distributed in an extended domain; e.g. the status of an advection flow in atmospheric circulation [22], the distribution of a dispersed population in Ecology [27] or the concentration of a chemical oscillator in a large vessel [28].

This work was funded by EU Marie Curie fellowship PIOF-GA-2009-235741. 
While easy to implement, CML were specifically designed to preclude numerical instabilities and other asymptotic divergences that materialize in traditional PDE's and coupled ODE's models [24]. Their simplicity and convenience contributed to their success as a simulation tool in many scientific disciplines [16]. This buoyant usage in applications has naturally called for the development of a mathematical theory that could put the observed phenomenology onto a rigorous footing.

Whereas their numerical simulations are easily accessible, the rigorous description of the global dynamics of CML is usually not [6]. The latent existence of large/infinite dimensional attractors brings additional problems to standard difficulties that arise in the analysis of concrete dynamical systems. Moreover, their mappings are typically composed of an individual nonlinearity at each lattice site and a (diffusive-like) interaction between the sites. The relative weight of these components is quantified by a coupling parameter whose intensity has a deep impact on the attractor; an increase of coupling may bring its dimension from a large number down to or below 1 .

Not surprisingly, mathematical results on arbitrarily large/infinite lattices have been limited to the cases where one of the ingredients dominates the dynamics. In particular, for (piecewise) expanding individual maps, accomplishments mostly concern the existence of physical measure in the weak interaction regime, when the CML resembles the interaction-free system, see e.g. $[1,4,5,13,19]$. In brief, these results are based on perturbation arguments applied to uniformly hyperbolic subsets. Proofs have however required substantial efforts and the introduction of original objects in order to be achieved for the models employed in simulations. For smooth real individual maps, some rigorous results can be found on synchrony phenomena that take place when the interaction prevails $[8,14]$. But to the best of our knowledge, no study exists on the (global) CML dynamics beyond the uncoupled regime when the individual map is (piecewise) expanding. Of note, few papers have considered the entire coupling range by limiting the analysis to small lattices, basically with 2 sites [10, 18, 20]. Moreover, proofs of the phase transitions, i.e. the co-existence of two 'Gibbs phases' on a unique indecomposable attractor, have been given in specially designed examples $[3,11]$.

The aim of this paper is to provide insights into the dynamics of (standard) CML on large lattices with piecewise expanding individual maps, throughout the domain where the mapping remains expanding. For simplicity, focus is on the description of repellers in periodic chains of coupled piecewise increasing Lorenz-type $[12,25,26]$ maps. The analysis follows the approach developed in the case of 2 sites $[7,9]$. It primarily consider piecewise linear maps and then extends the results by continuation to CML based on $C^{1}$-perturbations of the individual map.

Our strategy consists in analyzing the symbolic dynamics associated with the restriction of the CML to its repeller in the expanding regime. An equation for spatio-temporal symbolic sequences is established whose solutions coincide with the codes of orbits lying in the repeller. By analyzing 
the equation, the maximal coupling parameter up to which all sequences are solutions (and hence the CML remains conjugated to its interaction-free counterpart) is computed. Beyond that threshold (which does not depend on the chain length), many sequences are pruned and coupling-dependent estimates of both forbidden and admissible sequences are established. Inspired by the physical nature of diffusive-type interactions, the main criteria for (non-)admissibility is the size of space-time reactangles where the orbits are composed of heterogeneous configurations, viz. when their coordinates are out of sync.

The estimates imply upper and lower bounds on the topological entropy of repellers, which become sharper and sharper as the coupling is decreased back to the threshold. Not only these bounds apply to coupled periodic chains of any (even) length, but there are shown to be extensive, namely to scale linearly with the period length. In that way, we prove that while a macroscopic change of the dynamics occurs beyond the uncoupled regime, the dynamics remains "extensively" chaotic in a large part of the expanding domain.

\section{Model and main results}

We consider basic Coupled Map Lattices (CML) on periodic chains $\mathbb{Z}_{2 L}:=$ $\mathbb{Z} / 2 L \mathbb{Z}$ with even number $2 L$ of sites $\left(L \geqslant 1\right.$ is an arbitrary integer). ${ }^{1}$ Thus the phase space is the set $\mathbb{R}^{\mathbb{Z}_{2 L}}$ of periodic configurations $x=\left\{x_{s}\right\}_{s \in \mathbb{Z}_{2 L}}$ with real components $x_{s}$, endowed with uniform norm $\|\cdot\|$. In this set, the dynamics is generated by the iterations of the map $F_{\epsilon, 2 L}$ defined by $[6,16]$

$$
\left(F_{\epsilon, 2 L} x\right)_{s}=(1-\epsilon) f\left(x_{s}\right)+\frac{\epsilon}{2}\left(f\left(x_{s-1}\right)+f\left(x_{s+1}\right)\right), \forall s \in \mathbb{Z}_{2 L},
$$

where $\epsilon \in[0,1]$ is the coupling strength and the individual map $f$ is the piecewise affine symmetric expanding map [12, 25, 26], see Figure 1, left.

$$
f(\mathrm{x})=a \mathrm{x}-(a-1) H(\mathrm{x}-1 / 2), \forall \mathrm{x} \in \mathbb{R} .
$$

Here the slope $a>2$ is fixed and $H$ denotes the Heaviside function

$$
H(\mathrm{x})=\left\{\begin{array}{ll}
0 & \text { if } \quad \mathrm{x}<0 \\
1 & \text { if } \quad \mathrm{x} \geqslant 0
\end{array} \quad \forall \mathrm{x} \in \mathbb{R} .\right.
$$

The assumption $a>2$ implies that the repeller $k_{f}$ of the individual dynamical system $(\mathbb{R}, f)$, namely the subset of points $\mathrm{x} \in \mathbb{R}$ whose forward orbit $\left\{f^{t}(\mathrm{x})\right\}_{t \geqslant 0}$ is bounded, is a Cantor set bounded away from the discontinuity $\mathrm{x}=1 / 2$. As a consequence, $k_{f}$ is uniformly hyperbolic and hence structurally stable with respect to small $C^{1}$-perturbations. Moreover, the system $\left(k_{f}, f\right)$ is topologically conjugated to the full Bernoulli shift with two symbols and

\footnotetext{
${ }^{1}$ Assuming even number of sites makes the analysis much simpler. However, it is likely that the existence of extensive bounds on the topological entropy does not depend on that assumption and holds for periodic chains of any number of sites. Similarly, these bounds might hold for one-dimensional lattices with other types of boundary conditions instead of periodic ones.
} 

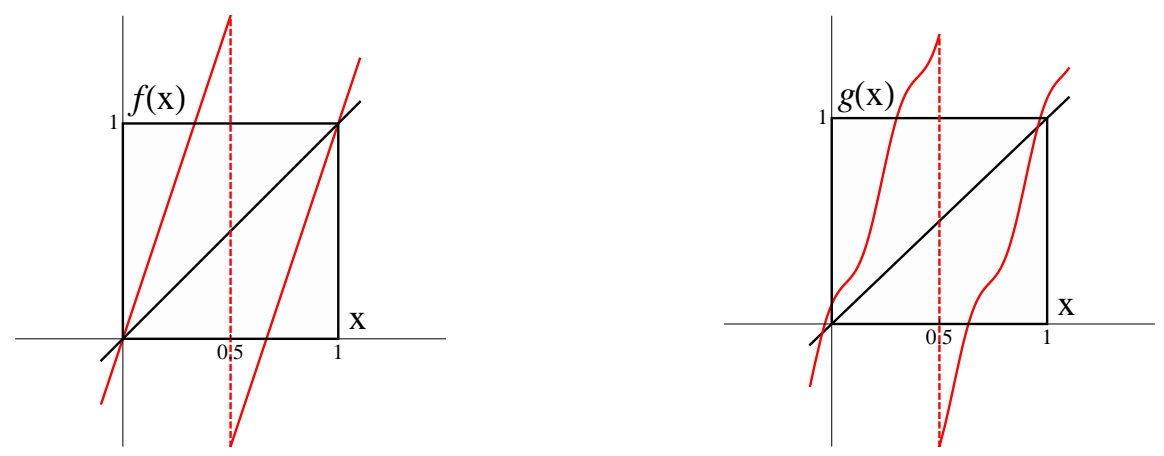

Figure 1. Left: Graph of the individual map $f$ for $a=3$.

Right: Graph of a small $C^{1}$-perturbation of $f$.

as such, its topological entropy is equal to $\log 2$. (For a definition and basic properties of the topological entropy in dynamical systems, see e.g. [17, 26].) In brief, the individual system is the most basic example of a simultaneously chaotic and robust system.

Given such simple features for the individual dynamics, we would like to get insights into the corresponding characteristics for the CML $\left(\mathbb{R}^{\mathbb{Z}_{2 L}}, F_{\epsilon, 2 L}\right)$. In particular, we ask about the topological entropy in the repeller $\mathcal{K}_{\epsilon, 2 L}$ (which, again, is defined as the set of points whose forward orbit remains bounded). For simplicity, we only consider the case where the CML is expanding, namely when $\epsilon \in\left[0, \epsilon_{e}\right]$ where $\epsilon_{e}=\frac{a-1}{2 a}$ is independent of $L$, see equation (12) in Appendix A.

Structural stability of the individual system implies that the same property holds for the uncoupled system $F_{0,2 L}$, uniformly in the lattice size [23]. As a consequence, $\left(\mathcal{K}_{\epsilon, 2 L}, F_{\epsilon, 2 L}\right)$ and $\left(\mathcal{K}_{0,2 L}, F_{0,2 L}\right)$ remain topologically conjugated provided that $\epsilon$ is small enough. Thanks to the piecewise affine assumption, the maximal coupling parameter up to which this property persists can be explicitly computed. When formulated in terms of the topological entropy $h_{\epsilon, 2 L}:=h_{\text {top }}\left(\mathcal{K}_{\epsilon, 2 L}, F_{\epsilon, 2 L}\right)$, the robustness of the uncoupled system with respect to small variations of coupling writes as follows.

Proposition 2.1. For every $L \geqslant 1$, we have $h_{\epsilon, 2 L}=h_{0,2 L}=(2 L) \log 2$ iff $\epsilon \leqslant \epsilon_{c}:=\frac{a-2}{2 a}$.

Notice that the maximal coupling parameter $\epsilon_{c}$ does not depend on $L$ and $\epsilon_{c}<\epsilon_{e}$. The proof of this statement relies on symbolic dynamics and is given in Section 3.2.

Since $(2 L) \log 2$ is a maximum for the CML entropy, Proposition 2.1 stipulates that this quantity must decrease beyond the uncoupled regime (i.e. $h_{\epsilon, 2 L}<(2 L) \log 2$ for all $L$ when $\epsilon>\epsilon_{c}$ ). However, it does not provide any estimate about the decay behavior when the number $L$ of sites diverges. A more accurate analysis of non-admissible sequences beyond $\epsilon_{c}$ shows that this decay is actually extensive. 
Proposition 2.2. For every $\epsilon>\epsilon_{c}$, there exists $\delta>0$ such that for all $L \geqslant 1$, we have $h_{\epsilon, 2 L}<(2 L)(\log 2-\delta)$.

For the proof, see Section 3.3. Of note, one expects that $\delta \rightarrow 0$ when $\epsilon \rightarrow \epsilon_{c}^{+}$. This property will follow from the next statement.

Whereas the CML entropy is subjected to extensive decay for $\epsilon>\epsilon_{c}$, it does not immediately drop off to zero. Uniform positive lower bounds exist for the entropy per lattice site $h_{\epsilon, 2 L} /(2 L)$ provided that $\epsilon$ is sufficiently close to the threshold $\epsilon_{c}$. More precisely, we have the following statement whose proof is given in Section 4.

Proposition 2.3. For every $0<\delta<\log 2$, there exists $\epsilon_{\delta}>\epsilon_{c}$ such that for all $\epsilon<\epsilon_{\delta}$ and $L \geqslant 1$, we have $h_{\epsilon, 2 L} \geqslant(2 L) \delta$.

This phenomenology, namely the existence of upper and lower bounds on the entropy per lattice site across the expanding coupling range, is not limited to piecewise affine CML. It extends to every CML with individual maps that are small $C^{1}$-perturbations of the piecewise affine map $f$, see Figure 1, right. In order to accurately state this result, let $g: \mathbb{R} \rightarrow \mathbb{R}$ be a continuous and increasing map for $\mathrm{x}<1 / 2$ and for $\mathrm{x} \geqslant 1 / 2$ and let

$$
a_{g}:=\inf _{\mathrm{x}<\mathrm{y}<\frac{1}{2} \text { or } \frac{1}{2} \leqslant \mathrm{x}<\mathrm{y}} \frac{g(\mathrm{y})-g(\mathrm{x})}{\mathrm{y}-\mathrm{x}} \text { and }\|g-f\|:=\sup _{\mathrm{x} \in \mathbb{R}}|g(\mathrm{x})-f(\mathrm{x})| .
$$

Let also $F_{g, \epsilon, 2 L}$ denote the CML with individual map $g$, namely

$$
\left(F_{g, \epsilon, 2 L} x\right)_{s}=(1-\epsilon) g\left(x_{s}\right)+\frac{\epsilon}{2}\left(g\left(x_{s-1}\right)+g\left(x_{s+1}\right)\right), \forall s \in \mathbb{Z}_{2 L} .
$$

Theorem 2.4. For every $0<\mu<\min \left\{\epsilon_{c},\left(\epsilon_{e}-\epsilon_{c}\right) / 2\right\}$, there exists $\eta>0$ such that for every $\epsilon<\epsilon_{e}-\mu$ and $L \geqslant 1$, any $C M L F_{g, \epsilon, 2 L}$ with individual map $g$ satisfying $\|g-f\|+\left|a_{g}-a\right|<\eta$ has a repeller $\mathcal{I}_{g, \epsilon, 2 L}$ on which the entropy satisfies

$$
\underline{\delta}_{\epsilon} \leqslant \frac{h_{t o p}\left(\mathcal{I}_{g, \epsilon, 2 L}, F_{g, \epsilon, 2 L}\right)}{2 L} \leqslant \bar{\delta}_{\epsilon},
$$

where the upper and lower bounds $\epsilon \mapsto \underline{\delta}_{\epsilon}$ and $\epsilon \mapsto \bar{\delta}_{\epsilon}$ have the following properties

$$
\left\{\begin{array}{c}
\underline{\delta}_{\epsilon}=\log 2 \quad \text { if } \quad \epsilon<\epsilon_{c}-\mu \\
\underline{\delta}_{\epsilon}<\log 2 \quad \text { if } \quad \epsilon>\epsilon_{c}-\mu
\end{array}, \lim _{\epsilon \rightarrow\left(\epsilon_{c}-\mu\right)^{+}} \underline{\delta}_{\epsilon}=\log 2,\right.
$$

and

$$
\begin{cases}\bar{\delta}_{\epsilon}=\log 2 & \text { if } \quad \epsilon<\epsilon_{c}+\mu \\ \bar{\delta}_{\epsilon}<\log 2 & \text { if } \quad \epsilon>\epsilon_{c}+\mu\end{cases}
$$

For the proof, see Section 5. A schematic illustration of the entropy bounds is given in Figure 2. 

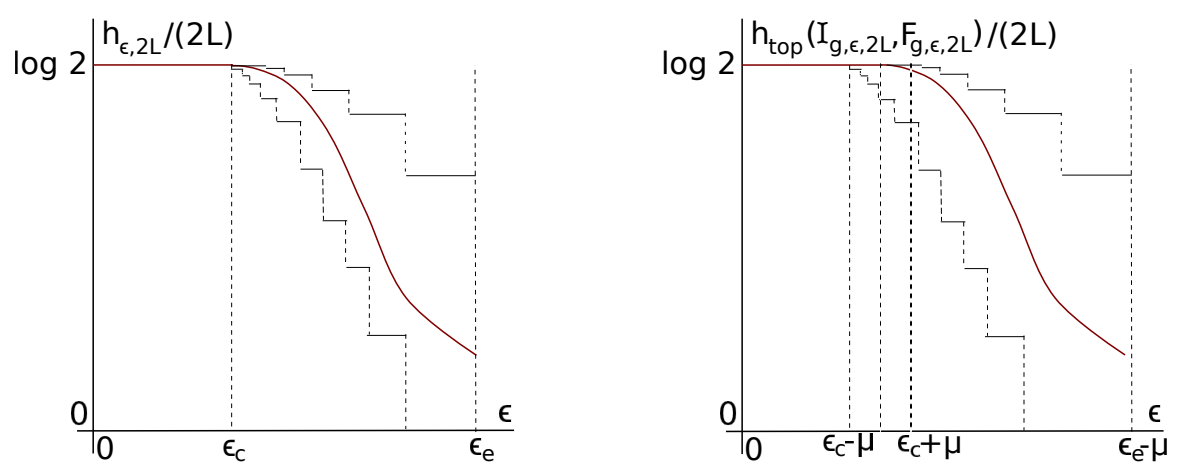

Figure 2. Schematic illustrations of upper and lower bounds (black step functions) on the CML entropy (red curves) per lattice site. Propositions 2.1, 2.2 and 2.3 and Theorem 2.4 attest that these bounds are independent of the lattice period $2 L$. Left: Piecewise affine CML $F_{\epsilon, 2 L}$. Right: CML $F_{g, \epsilon, 2 L}$ with piecewise increasing individual map $g$.

\section{Symbolic dynamics: Basic features}

\subsection{Symbolic description of the CML}

In order to define a symbolic dynamics for the CML, we first introduce a coding that is inspired from the natural coding of the individual map. Given a periodic configuration $x \in \mathbb{R}^{\mathbb{Z}_{2 L}}$, a periodic symbolic configuration $\left\{\theta_{s}\right\}_{s \in \mathbb{Z}_{2 L}} \in\{0,1\}^{\mathbb{Z}_{2 L}}$ is assigned according to the components location with respect to $1 / 2$. Namely, we set

$$
\theta_{s}=H\left(x_{s}-1 / 2\right), \forall s \in \mathbb{Z}_{2 L}
$$

where $H$ still denotes the Heaviside function. Now, the code $\theta=\left\{\theta^{t}\right\}_{t \geqslant 0}$ associated with $x$ is simply the sequence of symbolic configurations (the symbolic sequence for short) associated with the iterates $F_{\epsilon, 2 L}^{t} x$, viz.

$$
\theta_{s}^{t}=H\left(\left(F_{\epsilon, 2 L}^{t} x\right)_{s}-1 / 2\right), \forall s \in \mathbb{Z}_{2 L}, t \geqslant 0 .
$$

The symbolic dynamics of the CML relies on the property that configurations in the repeller can be entirely determined by their code. This is a consequence of Lemma 3.1 below. Moreover, their expression is known explicitly and depends upon the function $\chi_{\epsilon, 2 L}$ defined by

$$
\chi_{\epsilon, 2 L}(\theta)=\frac{a-1}{a} \sum_{k=0}^{+\infty} a^{-k} \sum_{n \in \mathbb{Z}_{2 L}} \ell_{n, 2 L}^{(k)} \theta_{n}^{k}
$$

where the coefficients $\ell_{n, 2 L}^{(k)}$ are the entries of the inverse powers $C_{\epsilon, 2 L}^{-k}$ of the coupling operator $C_{\epsilon, 2 L}$ (see Appendix A), namely we have

$$
\left(C_{\epsilon, 2 L}^{-k} x\right)_{s}=\sum_{n \in \mathbb{Z}_{2 L}} \ell_{n, 2 L}^{(k)} x_{s+n}, \forall s \in \mathbb{Z}_{2 L}
$$


for all $k \geqslant 0$ and $x \in \mathbb{R}^{\mathbb{Z}_{2 L}}$. This expression follows from the fact that $C_{\epsilon, 2 L}$ is a convolution operator on $\mathbb{R}^{\mathbb{Z}_{2 L}}$ and the inverse powers $C_{\epsilon, 2 L}^{-k}$ are well-defined when $\epsilon<\epsilon_{e}$ and are also convolution operators [1]. Throughout the paper, we shall extensively use various properties of the coefficients $\ell_{n, 2 L}^{(k)}$. These properties are collected in Appendix A. In particular, we show in Claim A.3 that

$$
\sum_{k=0}^{+\infty} a^{-k} \sum_{n \in \mathbb{Z}_{2 L}}\left|\ell_{n, 2 L}^{(k)}\right|<+\infty
$$

which implies that the function $\chi_{\epsilon, 2 L}$ is well-defined. Hence, not only this function provides an expression of configurations in the repeller but it can be used to characterize those symbolic sequences that are actually the codes of such configurations. Let $R_{\mathrm{sp}}$ and $R_{\mathrm{ti}}$ respectively denote the space and time translations acting on symbolic sequences, i.e.

$$
\left(R_{\mathrm{sp}} \theta\right)_{s}^{t}=\theta_{s+1}^{t} \quad \text { and } \quad\left(R_{\mathrm{ti}} \theta\right)_{s}^{t}=\theta_{s}^{t+1}, \forall s \in \mathbb{Z}_{2 L}, t \geqslant 0 .
$$

Lemma 3.1. A periodic configuration $x \in \mathbb{R}^{\mathbb{Z}_{2 L}}$ belongs to $\mathcal{K}_{\epsilon, 2 L}$ iff we have

$$
x_{s}=\chi_{\epsilon, 2 L} \circ R_{\mathrm{sp}}^{s}(\theta), \forall s \in \mathbb{Z}_{2 L}
$$

where $\theta$ is the code associated with $x$. Independently, given a symbolic sequence $\theta$, the configuration $\left\{\chi_{\epsilon, 2 L} \circ R_{\mathrm{sp}}^{s}(\theta)\right\}_{s \in \mathbb{Z}_{2 L}}$ belongs to $\mathcal{K}_{\epsilon, 2 L}$ iff $\theta$ solves the equation

$$
\theta_{s}^{t}=H\left(\chi_{\epsilon, 2 L} \circ R_{\mathrm{sp}}^{s} \circ R_{\mathrm{ti}}^{t}(\theta)-1 / 2\right), \forall s \in \mathbb{Z}_{2 L}, t \geqslant 0 .
$$

Notice that a version of this statement for $L=1$ has been given in [9].

Proof. We only show that if $x \in \mathcal{K}_{\epsilon, 2 L}$, then it writes $\left\{\chi_{\epsilon, 2 L} \circ R_{\mathrm{sp}}^{s}(\theta)\right\}_{s \in \mathbb{Z}_{2 L}}$. The proofs of other properties are direct and left to the reader.

The action of the mapping $F_{\epsilon, 2 L}$ can be written in an operator form as follows

$$
F_{\epsilon, 2 L} x=a C_{\epsilon, 2 L} x-(a-1) C_{\epsilon, 2 L} \theta^{0} .
$$

When $\epsilon<1 / 2$ (which is the case when $\epsilon<\epsilon_{e}$ ), all eigenvalues of $C_{\epsilon, 2 L}$ are positive and the smallest eigenvalue is $1-2 \epsilon$. Hence $C_{\epsilon, 2 L}$ is invertible and we have $\left\|C_{\epsilon, 2 L}^{-1}\right\|=(1-2 \epsilon)^{-1}$. By inverting the previous relation and iterating, one obtains the following expression

$$
x=a^{-t} C_{\epsilon, 2 L}^{-t} F_{\epsilon, 2 L}^{t} x+(a-1) \sum_{k=0}^{t-1} a^{-(k+1)} C_{\epsilon, 2 L}^{-k} \theta^{k}, \forall t \geqslant 0 .
$$

If the configuration $x \in \mathcal{K}_{\epsilon, 2 L}$, then the definition of this set implies that the norm $\left\|F_{\epsilon, 2 L}^{t} x\right\|$ remains bounded for all times. The assumption $\epsilon<\epsilon_{e}$ then yields

$$
\lim _{t \rightarrow+\infty}\left\|a^{-t} C_{\epsilon, 2 L}^{-t} F_{\epsilon, 2 L}^{t} x\right\|=0 .
$$


Hence by taking the limit $t \rightarrow+\infty$ in the expression of $x$ above, we obtain the following expression for its components

$$
x_{s}=\frac{a-1}{a} \sum_{k=0}^{+\infty} a^{-k}\left(C_{\epsilon, 2 L}^{-k} \theta^{k}\right)_{s}, \forall s \in \mathbb{Z}_{2 L}
$$

which is the desired expression.

Every solution of the admissibility equation (4) is called an admissible (symbolic) sequence. Let $\mathcal{A}_{\epsilon, 2 L}$ be the set of solutions, endowed with the product topology (of discrete topology).

Lemma 3.1 can be interpreted as the existence of a conjugacy, i.e. a uniformly continuous bijection, ${ }^{2}$ between the symbolic system $\left(\mathcal{A}_{\epsilon, 2 L}, R_{\mathrm{ti}}\right)$ and the CML $\left(\mathcal{K}_{\epsilon, 2 L}, F_{\epsilon, 2 L}\right)$. In general, this conjugacy needs not be an homeomorphism and the topological entropies of the two systems need not be equal - because $\mathcal{A}_{\epsilon, 2 L}$ may not be compact. However, uniform continuity and monotonicity of the entropy with respect to set inclusion assert the inequalities (see e.g. Proposition 3.1.6 and its proof in [17])

$$
\sup _{A \subset \mathcal{A}_{\epsilon, 2 L}, A \text { compact, invariant }} h_{\mathrm{top}}\left(A, R_{\mathrm{ti}}\right) \leqslant h_{\epsilon, 2 L} \leqslant h_{\mathrm{top}}\left(\mathcal{A}_{\epsilon, 2 L}, R_{\mathrm{ti}}\right), \forall \epsilon<\epsilon_{e},
$$

where $h_{\mathrm{top}}\left(A, R_{\mathrm{ti}}\right)$ and $h_{\mathrm{top}}\left(\mathcal{A}_{\epsilon, 2 L}, R_{\mathrm{ti}}\right)$ denote the entropy of the symbolic (sub-)systems.

\subsection{The uncoupled regime $\epsilon<\epsilon_{c}$}

Based on Lemma 3.1, our strategy to investigate topological properties of the CML dynamics is to determine which symbolic sequences are solutions of the admissibility equation depending on the coupling parameter. Here, we consider the simplest case where all sequences are admissible and we rely on basic properties of the coefficients $\ell_{n, 2 L}^{(k)}$ in $\chi_{\epsilon, 2 L}$ to compute the maximal parameter up to which this property holds.

Lemma 3.2. All symbolic sequences are admissible iff $\epsilon<\epsilon_{c}:=\frac{a-2}{2 a}$.

Proof. A sequence $\theta$ solves the admissibility equation (4) iff

$$
\begin{aligned}
& \chi_{\epsilon, 2 L} \circ R_{\mathrm{sp}}^{s} \circ R_{\mathrm{ti}}^{t}(\theta)<1 / 2 \text { if } \theta_{s}^{t}=0 \\
& \chi_{\epsilon, 2 L} \circ R_{\mathrm{sp}}^{s} \circ R_{\mathrm{ti}}^{t}(\theta) \geqslant 1 / 2 \text { if } \theta_{s}^{t}=1
\end{aligned} \forall s \in \mathbb{Z}_{2 L}, t \geqslant 0 .
$$

Therefore, all symbolic sequences are admissible when the following inequalities hold

$$
\sup _{\theta \in \Omega_{2 L}: \theta_{0}^{0}=0} \chi_{\epsilon, 2 L}(\theta)<1 / 2 \text { and } \inf _{\theta \in \Omega_{2 L}: \theta_{0}^{0}=1} \chi_{\epsilon, 2 L}(\theta) \geqslant 1 / 2 .
$$

where $\Omega_{2 L}$ is the set of all symbolic sequences, viz.

$$
\Omega_{2 L}:=\left\{\theta=\left\{\theta_{s}^{t}\right\}_{s \in \mathbb{Z}_{2 L}, t \geqslant 0},: \theta_{s}^{t} \in\{0,1\}\right\} .
$$

\footnotetext{
${ }^{2}$ We use the term conjugacy when the correspondence is a uniformly continuous bijection and topological conjugacy when the map is a homeomorphism.
} 
By continuity of the function $\theta \mapsto \chi_{\epsilon, 2 L}(\theta)$ and compactness of $\Omega_{2 L}$, these upper and lower bounds are attained. Furthermore, the normalization

$$
\sum_{n \in \mathbb{Z}_{2 L}} \ell_{n, 2 L}^{(k)}=1 \forall k \geqslant 0
$$

(see Claim A.2 in Appendix A) implies that following symmetry holds

$$
\chi_{\epsilon, 2 L}(1-\theta)=1-\chi_{\epsilon, 2 L}(\theta)
$$

where $1-\theta:=\left\{1-\theta_{s}^{t}\right\}_{s \in \mathbb{Z}_{2 L}, t \geqslant 0}$. Consequently, the previous conditions are equivalent to

$$
\sup _{\theta \in \Omega_{2 L}: \theta_{0}^{0}=0} \chi_{\epsilon, 2 L}(\theta)<1 / 2 .
$$

Now, using the properties $\ell_{n, 2 L}^{(0)}=\delta_{n, 0 \bmod 2 L}$ and $\ell_{2 n, 2 L}^{(k)}>0$ and $\ell_{2 n+1,2 L}^{(k)}<0$ (see Lemma A.1 in Appendix A again) in the expression of $\chi_{\epsilon, 2 L}$, it easily follows that

$$
\sup _{\theta \in \Omega_{2 L}: \theta_{0}^{0}=0} \chi_{\epsilon, 2 L}(\theta)=\chi_{\epsilon, 2 L}\left(\theta^{0}\left[(10)^{L}\right]^{\infty}\right)
$$

where $\theta_{0}^{0}=0$ and $\theta_{s}^{0}$ is arbitrary for $s \neq 0 \bmod 2 L$. Moreover $\left[(10)^{L}\right]^{\infty}$ is a shortcut for

$$
\theta_{s}^{t}=H\left((-1)^{s}\right), \forall s \in \mathbb{Z}_{2 L}, t \geqslant 1
$$

In particular, the maximum is attained for a sequence of 2-periodic symbolic configurations (hence it does not depend on $L$ ), viz.

$$
\begin{aligned}
\chi_{\epsilon, 2 L}\left(\theta^{0}\left[(10)^{L}\right]^{\infty}\right) & =\chi_{\epsilon, 2}\left(\left[0 \theta_{1}^{0}\right][10]^{\infty}\right)=\frac{a-1}{a} \sum_{k=1}^{+\infty} a^{-k} \ell_{0,2}^{(k)} \\
& =\frac{a-1}{2 a}\left(\frac{1}{a-1}+\frac{1}{b_{\epsilon}-1}\right) .
\end{aligned}
$$

where we have used the explicit expression of $\ell_{0,2}^{(k)}$ from equation (15) in Appendix $\mathrm{A}$ and $b_{\epsilon}:=a(1-2 \epsilon)$. It immediately follows that the condition (6) holds iff $b_{\epsilon}>2$, i.e. iff $\epsilon<\epsilon_{c}$.

Proof of Proposition 2.1. Lemma 3.2 states that $\mathcal{A}_{\epsilon}^{(2 L)}=\Omega_{2 L}$ when $\epsilon<$ $\epsilon_{c}$. Since this set is compact, the conjugacy between the symbolic system $\left(\mathcal{A}_{\epsilon, 2 L}, R_{\mathrm{ti}}\right)$ and the $\mathrm{CML}\left(\mathcal{K}_{\epsilon, 2 L}, F_{\epsilon, 2 L}\right)$ is a homeomorphism. Hence, we have $h_{\epsilon, 2 L}=h_{\mathrm{top}}\left(\Omega_{2 L}, R_{\mathrm{ti}}\right)=(2 L) \log 2$ is maximal in this domain. The equality $h_{\epsilon_{c}, 2 L}=(2 L) \log 2$ will be shown in the proof of Proposition 2.3 below.

On another hand, the end of the proof of Lemma 3.2 shows that

$$
\chi_{\epsilon, 2 L}\left(\theta^{0}\left[(10)^{L}\right]^{\infty}\right)>1 / 2
$$

for every sequence with $\theta_{0}^{0}=0$ when $\epsilon>\epsilon_{c}$. By continuity of the map $\theta \mapsto \chi_{\epsilon, 2 L}(\theta)$, there exists $T_{\epsilon, 2 L} \geqslant 1$ such that for any sequence $\theta$ so that

$$
\theta_{0}^{0}=0 \text { and } \theta^{t}=(10)^{L} 1 \leqslant t \leqslant T_{\epsilon, 2 L}
$$

we have $\chi_{\epsilon, 2 L}(\theta)>1 / 2$; hence $\theta$ is not admissible. By a standard argument that we detail below, this implies that $h_{\mathrm{top}}\left(\mathcal{A}_{\epsilon}^{(2 L)}, R_{\mathrm{ti}}\right)<(2 L) \log 2$ which, 
together with the inequality (5), implies that $h_{\epsilon, 2 L}<(2 L) \log 2$ when $\epsilon>\epsilon_{c}$, as desired.

The remaining argument relies on the fact that the topological entropy of the symbolic subshift $\left(\mathcal{A}_{\epsilon, 2 L}, R_{\mathrm{ti}}\right)$ can be characterized as follows $[17,21]$

$$
h_{\mathrm{top}}\left(\mathcal{A}_{\epsilon, 2 L}, R_{\mathrm{ti}}\right)=\lim _{T \rightarrow \infty} \frac{\log N_{T, 2 L}}{T}=\inf _{T \geqslant 1} \frac{\log N_{T, 2 L}}{T} .
$$

where $N_{T, 2 L}$ is the number of admissible words of length $T$ in $\mathcal{A}_{\epsilon, 2 L}$. In particular, we must have $h_{\text {top }}\left(\mathcal{A}_{\epsilon, 2 L}, R_{\mathrm{ti}}\right) \leqslant \frac{\log N_{T, 2 L}}{T}$ for every $T \geqslant 1$.

In the present case, the non-admissibility of the sequences that satisfy relation (7) and of symmetric sequences imply the following inequality

$$
N_{T_{\epsilon, 2 L}+1,2 L} \leqslant 2^{(2 L)\left(T_{\epsilon, 2 L}+1\right)}-2^{2 L}
$$

which yields

$$
h_{\mathrm{top}}\left(\mathcal{A}_{\epsilon, 2 L}, R_{\mathrm{ti}}\right) \leqslant \frac{\log N_{T_{\epsilon, 2 L}+1,2 L}}{T_{\epsilon, 2 L}+1}<\frac{(2 L)\left(T_{\epsilon, 2 L}+1\right)}{T_{\epsilon, 2 L}+1} \log 2=(2 L) \log 2 .
$$

The proof is complete.

For $\epsilon>\epsilon_{c}$, it is reasonable to expect that the entropy $h_{\epsilon, 2 L}$ would monotonically decrease with $\epsilon$. While this property remains to be proved, a similar trait can be shown for the symbolic system. This is an immediate consequence of the fact that admissibility of symbolic sequences is a monotonic property in a neighborhood of $\epsilon_{c}$, namely

$$
\mathcal{A}_{\epsilon_{1}, 2 L} \supset \mathcal{A}_{\epsilon_{2}, 2 L},
$$

when $\epsilon_{1}<\epsilon_{2}$ (are sufficiently close to $\epsilon_{c}$ ). The proof is identical to the one of Proposition 3.2 in [9] and essentially relies on the following property (which in turn is a consequence of the fact that $\ell_{L, 2 L}^{(k)}$ is the entry with smallest modulus among the configuration $\left\{\ell_{n, 2 L}^{(k)}\right\}_{n \in \mathbb{Z}_{2 L}}$ )

$$
\sup _{\theta^{0} \in\{0,1\}^{\mathbb{Z}_{2 L}}: \theta^{0} \neq(10)^{L}}\left(C_{\epsilon, 2 L}^{-k} \theta^{0}\right)_{0}=\left(C_{\epsilon, 2 L}^{-k} \tilde{\theta}^{0}\right)_{0}, \forall k \geqslant 1,
$$

where $\tilde{\theta}_{s}^{0}=H\left((-1)^{s}\right)$ for all $s \in \mathbb{Z}_{2 L}$, except for $s=L \bmod 2 L$. The details are left to the reader.

\subsection{Finite spatio-temporal word non-admissibility when $\epsilon>\epsilon_{c}$}

As shown in the end of the proof of Proposition 2.1 above, beyond the uncoupled regime, the CML entropy must be smaller than its maximal value for every even number of sites, viz. $h_{\epsilon, 2 L}<(2 L) \log 2$ for all $L \geqslant 1$. In order to obtain a non-trivial uniform upper bound for the entropy per lattice site, one needs to refine the restriction (7) on admissible sequences. One way to proceed is to demonstrate that the presence of specific (large) spatio-temporal motifs in a sequence induces non-admissibility. This is the purpose of the next statement. 


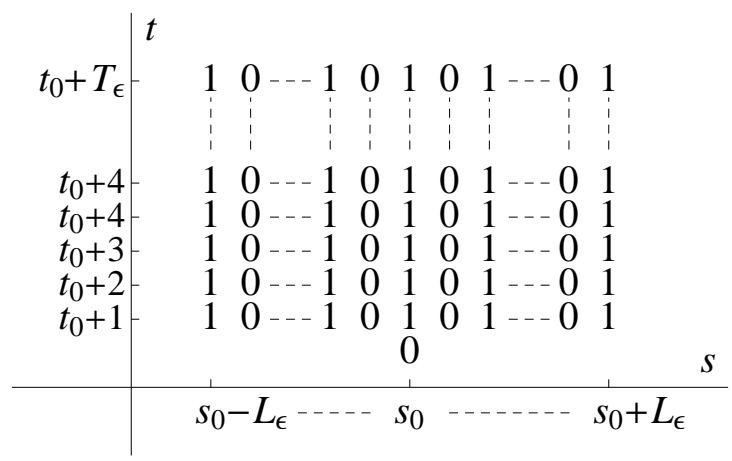

Figure 3. Graphic representation of the first forbidden word in Lemma 3.3. The other word is obtained by exchanging the 0 's and the 1 's.

Lemma 3.3. For every $\epsilon>\epsilon_{c}$, there exist $L_{\epsilon}, T_{\epsilon} \geqslant 1$ such that for every $L>L_{\epsilon}$, every symbolic sequence $\theta \in \Omega_{2 L}$ for which there exists $\left(s_{0}, t_{0}\right)$ such that (see Figure 3)

$$
\left\{\begin{array}{l}
\text { either } \theta_{s_{0}}^{t_{0}}=0 \text { and } \theta_{s_{0}+s}^{t_{0}+t}=H\left((-1)^{s}\right) \\
\text { or } \theta_{s_{0}}^{t_{0}}=1 \text { and } \theta_{s_{0}+s}^{t_{0}+t}=H\left((-1)^{s+1}\right)
\end{array} \quad \forall-L_{\epsilon} \leqslant s<L_{\epsilon}, 1 \leqslant t \leqslant T_{\epsilon}\right.
$$

is not admissible.

Proof. We begin to prove that if two symbolic sequences coincide on a sufficiently large rectangular space-time window that contains the origin, then their image under $\chi_{\epsilon, 2 L}$ are arbitrarily close to each other. Given $L, L^{\prime}, T \geqslant 1$ with $L^{\prime}<L$, let $\theta, \tilde{\theta} \in \Omega_{2 L}$ be two sequences such that

$$
\theta_{s}^{t}=\tilde{\theta}_{s}^{t}, \forall-L^{\prime} \leqslant s<L^{\prime}, 0 \leqslant t \leqslant T \text {. }
$$

Since we have $\left|\theta_{s}^{t}-\tilde{\theta}_{s}^{t}\right| \leqslant 1$ for the remaining pairs $(s, t)$, it follows from the definition of the function $\chi_{\epsilon, 2 L}$ above that

$$
\left|\chi_{\epsilon, 2 L}(\theta)-\chi_{\epsilon, 2 L}(\tilde{\theta})\right| \leqslant \bar{m}_{T}+\underline{m}_{L^{\prime}, L, T}
$$

where

$$
\begin{array}{r}
\bar{m}_{T}=\frac{a-1}{a} \sum_{k=T+1}^{+\infty} a^{-k} \sum_{n \in \mathbb{Z}_{2 L}}\left|\ell_{n, 2 L}^{(k)}\right| \text { and } \\
\underline{m}_{L^{\prime}, L, T}=\frac{a-1}{a} \sum_{k=0}^{T} a^{-k} \sum_{-L \leqslant n<-L^{\prime} \text { or } L^{\prime} \leqslant n<L}\left|\ell_{n, 2 L}^{(k)}\right| .
\end{array}
$$

The first term $\bar{m}_{T}$ is estimated using the property $\sum_{n \in \mathbb{Z}_{2 L}}\left|\ell_{n, 2 L}^{(k)}\right|=(1-2 \epsilon)^{-k}$ (Claim A.3 in Appendix A - recall that $b_{\epsilon}=a(1-2 \epsilon)$ )

$$
\bar{m}_{T}=\frac{a-1}{a} \sum_{k=T+1}^{+\infty} a^{-k}\left(\ell_{0,2}^{(k)}-\ell_{1,2}^{(k)}\right)=\frac{a-1}{a} \frac{b_{\epsilon}^{-T}}{b_{\epsilon}-1} .
$$


In particular, since $b_{\epsilon}>1$ when $\epsilon<\epsilon_{e}$, we have $\lim _{T \rightarrow+\infty} \bar{m}_{T}=0$.

In order to control the second term $\underline{m}_{L^{\prime}, L, T}$, we consider the entries $\left\{\ell_{n}^{(k)}\right\}_{n \in \mathbb{Z}}$ of the inverse powers $C_{\epsilon}^{-k}$ of the coupling operator $C_{\epsilon}$ defined on bounded configurations of the infinite lattice $\mathbb{Z}$. (Just as for its periodic version, the operator $C_{\epsilon}$ is invertible when $\epsilon<\epsilon_{e}$ and its inverse powers are all convolution operators [1].) That is to say, given $x \in \ell^{\infty}(\mathbb{Z})$ we have

$$
\left(C_{\epsilon}^{-k} x\right)_{s}=\sum_{n \in \mathbb{Z}} \ell_{n}^{(k)} x_{s+n} \forall s \in \mathbb{Z}
$$

The coefficients $\ell_{n}^{(k)}$ have the same sign properties as the $\ell_{n, 2 L}^{(k)}$ in Lemma A.1, see relation (16) in Appendix A. Moreover we have

$$
\ell_{n, 2 L}^{(k)}=\sum_{m \in \mathbb{Z}} \ell_{n+m(2 L)}^{(k)}, \forall n \in \mathbb{Z}_{2 L}
$$

This easily implies the following inequality

$$
\sum_{-L \leqslant n<-L^{\prime} \text { or } L^{\prime} \leqslant n<L}\left|\ell_{n, 2 L}^{(k)}\right| \leqslant \sum_{-L \leqslant n<-L^{\prime} \text { or } L^{\prime} \leqslant n<L}\left|\ell_{n}^{(k)}\right|=\left(C_{\epsilon}^{-k} x_{L^{\prime}}\right)_{0}
$$

where the infinite configuration $x_{L^{\prime}}$ is defined by (see Figure 4 )

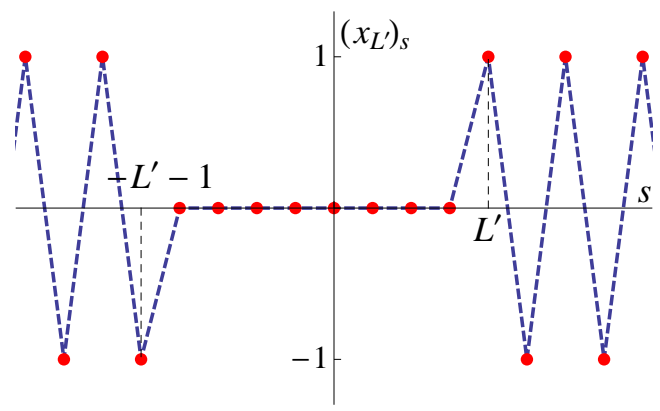

FiguRE 4. Graphic representation of the configuration $x_{L^{\prime}}$ defined in the proof of Lemma 3.3.

$$
\left(x_{L^{\prime}}\right)_{s}=\left(H\left(s-L^{\prime}\right)+H\left(-s-L^{\prime}-1\right)\right)(-1)^{s}, \forall s \in \mathbb{Z} .
$$

We have $\left\|x_{L^{\prime}}\right\|=1$ for all $L^{\prime} \geqslant 1$ and the sequence $\left\{x_{L^{\prime}}\right\}_{L^{\prime} \geqslant 1}$ point-wise converges to 0 (i.e. the configuration defined by $x_{s}=0$ for all $s \in \mathbb{Z}$ ) as $L^{\prime} \rightarrow+\infty$. Convolution operators commute with point-wise limits of equibounded sequences [1]. It results that for every $k \geqslant 0$, we have

$$
\lim _{L^{\prime} \rightarrow+\infty}\left(C_{\epsilon}^{-k} x_{L^{\prime}}\right)_{0}=\left(C_{\epsilon}^{-k} 0\right)_{0}=0
$$

which yields $\lim _{L^{\prime}, L \rightarrow+\infty} \underline{m}_{L^{\prime}, L, T}=0$ for every $T \geqslant 1$. This completes the first part of the proof.

Now given $\epsilon>\epsilon_{c}$, let $\eta=\chi_{\epsilon, 2}\left(\left[0 \theta_{1}^{0}\right][10]^{\infty}\right)-1 / 2>0$ where $\theta_{1}^{0}$ is arbitrary (see end of the proof of Lemma 3.2). According to the first part of the proof, 
let $T_{\epsilon}$ be sufficiently large such that $\bar{m}_{T_{\epsilon}}<\eta / 2$. Then, let $L_{\epsilon}$ be large enough such that $\underline{m}_{L_{\epsilon}, L, T_{\epsilon}}<\eta / 2$ for every $L>L_{\epsilon}$.

By construction, any sequence $\theta \in \Omega_{2 L}$ where $\theta_{0}^{0}=0$ and $\theta_{s}^{t}=H\left((-1)^{s}\right)$ for $|s|<L_{\epsilon}$ and $1 \leqslant t \leqslant T_{\epsilon}$ is such that

$$
\left|\chi_{\epsilon, 2 L}(\theta)-\chi_{\epsilon, 2}\left(\left[0 \theta_{1}^{0}\right][10]^{\infty}\right)\right| \leqslant \bar{m}_{T_{\epsilon}}+\underline{m}_{L_{\epsilon}, L, T_{\epsilon}}<\eta
$$

and hence $\chi_{\epsilon, 2 L}(\theta)>1 / 2$. Since $\theta_{0}^{0}=0$, this shows that this sequence can not be admissible. By symmetry, one proves that any sequence such that $\theta_{0}^{0}=1$ and $\theta_{s}^{t}=H\left((-1)^{s+1}\right)$ for $|s|<L_{\epsilon}$ and $1 \leqslant t \leqslant T_{\epsilon}$ is also not admissible. Finally, for any symbolic sequence $\theta$ as in the statement, the space-time translated sequence $R_{\mathrm{sp}}^{s_{0}} \circ R_{\mathrm{ti}}^{t_{0}}(\theta)$ must be of one of the previous types. As such, $\theta$ cannot be admissible and the proof is complete.

Proof of Proposition 2.2. Here $L_{\epsilon}$ and $T_{\epsilon}$ refer to the integers that were defined in Lemma 3.3. We are going to prove that this Lemma implies the existence of $\delta_{1}, \delta_{2}>0$ for every $\epsilon>\epsilon_{c}$ such that

$$
h_{\text {top }}\left(\mathcal{A}_{\epsilon, 2 L}, R_{\mathrm{ti}}\right) \leqslant 2 L\left(\log 2-\delta_{1}\right)+\delta_{2}, \forall L>L_{\epsilon} .
$$

Then letting $\tilde{L}$ be sufficiently large such that $\tilde{\delta}_{1}:=\delta_{1}-\delta_{2} /(2 \tilde{L})>0$ and using from Proposition 2.1 that $\tilde{\delta}_{2}:=\log 2-\max _{1 \leqslant L<\tilde{L}} \frac{h_{\epsilon, 2 L}}{2 L}>0$, the Proposition easily follows with $\delta=\min \left\{\tilde{\delta}_{1}, \tilde{\delta}_{2}\right\}$.

Recall from the proof of Proposition 2.1 that we must have $h_{\mathrm{top}}\left(\mathcal{A}_{\epsilon, 2 L}, R_{\mathrm{ti}}\right) \leqslant$ $\frac{\log N_{T_{\epsilon}+1,2 L}}{T_{\epsilon}+1}$ where $N_{T, 2 L}$ denotes the number of admissible words of length $T$ in $\mathcal{A}_{\epsilon, 2 L}$.

Let now $L>L_{\epsilon}$. A word $\left\{\theta_{s}^{t}\right\}_{s \in \mathbb{Z}_{2 L}}^{t=0, \cdots, T_{\epsilon}}$ can be admissible only if each (nonoverlapping) restriction on spatial strips of width $2 L_{\epsilon}$ namely

$$
\left\{\theta_{s}^{t}\right\}_{s=r\left(2 L_{\epsilon}\right), \cdots,(r+1)\left(2 L_{\epsilon}\right)-1}^{t=0, \cdots, T_{\epsilon}}, \forall r=0, \cdots,\left\lfloor\frac{L}{L_{\epsilon}}\right\rfloor-1
$$

(where $\lfloor\cdot\rfloor$ denotes the floor function) is distinct from any forbidden motif given in Lemma 3.3. It means that if $\theta_{r\left(2 L_{\epsilon}\right)+L_{\epsilon}}^{0}=0$ then there must be $-L_{\epsilon} \leqslant s<L_{\epsilon}$ and $1 \leqslant t \leqslant T_{\epsilon}$ such that

$$
\theta_{r\left(2 L_{\epsilon}\right)+L_{\epsilon}+s}^{t} \neq H\left((-1)^{s}\right)
$$

and similarly if $\theta_{r\left(2 L_{\epsilon}\right)+L_{\epsilon}}^{0}=1$.

Notice that, given $\theta_{r\left(2 L_{\epsilon}\right)+L_{\epsilon}}^{0}$ in such motifs, the other symbols for $t=0$ and $-L_{\epsilon} \leqslant s<L_{\epsilon}, s \neq 0$ are arbitrary. Hence there are $2^{2 L_{\epsilon}-1}$ motifs for each choice of $\theta_{r\left(2 L_{\epsilon}\right)+L_{\epsilon}}^{0}$ and thus there are $2^{2 L_{\epsilon}}$ forbidden motifs for each $r$.

Consequently, there remain at most $2^{2 L_{\epsilon}\left(T_{\epsilon}+1\right)}-2^{2 L_{\epsilon}}$ choices of admissible motifs on each strip $s=r\left(2 L_{\epsilon}\right), \cdots,(r+1)\left(2 L_{\epsilon}\right)-1$. Therefore, there are at most $\left(2^{2 L_{\epsilon}\left(T_{\epsilon}+1\right)}-2^{2 L_{\epsilon}}\right)^{\left\lfloor\frac{L}{L_{\epsilon}}\right\rfloor}$ admissible sub-words on the strip $s=0, \cdots,\left\lfloor\frac{L}{L_{\epsilon}}\right\rfloor L_{\epsilon}-$ 1. Assuming maximal diversity for the remaining sites $s=\left\lfloor\frac{L}{L_{\epsilon}}\right\rfloor L_{\epsilon}, \cdots, L-1$, we finally obtain the following upper bound

$$
N_{T_{\epsilon}+1,2 L} \leqslant\left(2^{2 L_{\epsilon}\left(T_{\epsilon}+1\right)}-2^{2 L_{\epsilon}}\right)^{\left\lfloor\frac{L}{L_{\epsilon}}\right\rfloor} 2^{\left(L-\left\lfloor\frac{L}{L_{\epsilon}}\right\rfloor L_{\epsilon}\right)\left(T_{\epsilon}+1\right)}
$$


from which, using basic inequalities for the floor function (viz. $x-1<\lfloor x\rfloor \leqslant x$ for all $x \in \mathbb{R}$ ), it easily follows that

$$
\frac{\log N_{T_{\epsilon}+1,2 L}}{T_{\epsilon}+1} \leqslant 2 L\left(\log 2-\delta_{1}\right)+\delta_{2}
$$

as desired, where $\delta_{1}=-\frac{\log \left(1-2^{-2 L_{\epsilon} T_{\epsilon}}\right)}{2 L_{\epsilon}\left(T_{\epsilon}+1\right)}>0$ and $\delta_{2}=L_{\epsilon} \log 2$.

\section{Families of admissible subshifts with positive entropy per lattice unit}

The strategy of the proof of Proposition 2.3 consists in showing the admissibility of certain symbolic subshifts that are simultaneously compact and with topological entropy equal to $(2 L) \delta_{\epsilon}$ where $\delta_{\epsilon}>0$ approaches $\log 2$ as $\epsilon \rightarrow \epsilon_{c}^{+}$. Compactness implies the lower bound $h_{\epsilon, 2 L} \geqslant(2 L) \delta_{\epsilon}$ as desired. More precisely, we introduce a one-parameter family of nested subshifts with appropriate entropy in the limit of large lattices, and we prove couplingdependent admissibility for every set in this family.

The subshift definition relies on the following terminology. The symbolic configurations $0^{2 L}$ and $1^{2 L}$ are called homogeneous configurations. Any other configuration is said to be heterogeneous. Now, given a configuration $\left\{\theta_{s}\right\}:=$ $\left\{\theta_{s}\right\}_{s \in \mathbb{Z}_{2 L}}$, let the function $\sigma:\{0,1\}^{\mathbb{Z}_{2 L}} \mapsto\{0,1\}$ be defined by

$$
\sigma\left(\left\{\theta_{s}\right\}\right)=\left\{\begin{array}{l}
0 \text { if }\left\{\theta_{s}\right\} \text { is homogeneous } \\
1 \text { if }\left\{\theta_{s}\right\} \text { is heterogeneous }
\end{array}\right.
$$

The idea behind the definition of the subshifts is to restrict repeated occurrences of heterogeneous configurations. Recall that symbolic sequences are denoted by $\theta=\left\{\theta^{t}\right\}_{t \geqslant 0}$ where each $\theta^{t}$ is a symbolic configuration. Given $\nu \in[0,1]$, let

$$
\Omega_{\nu, 2 L}=\left\{\theta \in \Omega_{2 L}: \sum_{t=t_{1}}^{t_{2}-1} \sigma\left(\theta^{t}\right) \leqslant\left\lceil\nu\left(t_{2}-t_{1}\right)\right\rceil, \forall 0 \leqslant t_{1}<t_{2}\right\}
$$

where $\lceil\cdot\rceil$ is the ceiling function. Notice that $\Omega_{1,2 L}$ contains all symbolic sequences, $\Omega_{0,2 L}$ only consists of homogeneous sequences and $\Omega_{\nu_{1}, 2 L} \subsetneq \Omega_{\nu_{2}, 2 L}$ when $\nu_{1}<\nu_{2}$. Moreover, we shall prove below that the sequence

$$
\left\{h_{\text {top }}\left(\Omega_{\frac{n}{n+1}, 2 L}, R_{\mathrm{ti}}\right)\right\}_{n \geqslant 0}
$$

(regarded as a sequence of sequences indexed by $L$ ) uniformly converges to $\{(2 L) \log 2\}_{L \geqslant 1}$. Together with the following statement, this property suffices to ensure that Proposition 2.3 holds. Note that the proof of Lemma 4.2 proves that each sequence $\left\{h_{\text {top }}\left(\Omega_{\frac{n}{n+1}}, 2 L, R_{\mathrm{ti}}\right)\right\}_{n \geqslant 0}$ is strictly increasing. We believe that the function $\nu \mapsto h_{\text {top }}\left(\Omega_{\nu, 2 L}, R_{\mathrm{ti}}\right)$ is actually strictly increasing.

Lemma 4.1. (i) For every $\epsilon>\epsilon_{c}$, there exists $\nu_{\epsilon} \in[0,1)$ such that for every $\nu<\nu_{\epsilon}$ we have

$$
\Omega_{\nu, 2 L} \subset \mathcal{A}_{\epsilon, 2 L}, \forall L \geqslant 1
$$


Moreover, for every $\nu>\nu_{\epsilon}$ and $L \geqslant 1$, some of the sequences in $\Omega_{\nu, 2 L}$ are not admissible.

(ii) The map $\epsilon \mapsto \nu_{\epsilon}$ is a decreasing Devil's staircase with limit $\lim _{\epsilon \rightarrow \epsilon_{c}^{+}} \nu_{\epsilon}=$ 1 .

Proof. Since each set $\Omega_{\nu, 2 L}$ is compact and is invariant under the action of $\theta \mapsto 1-\theta$, the reasoning at the beginning of the proof of Lemma 3.2 can be repeated here to conclude that $\Omega_{\nu, 2 L} \subset \mathcal{A}_{\epsilon, 2 L}$ iff

$$
\sup _{\theta \in \Omega_{\nu, 2 L}: \theta_{0}^{0}=0} \chi_{\epsilon, 2 L}(\theta)<1 / 2
$$

The strategy to determine the optimal $\nu_{\epsilon}$ is to obtain a manageable expression for this supremum. Recall that the definition of $\chi_{\epsilon, 2 L}$, namely

$$
\chi_{\epsilon, 2 L}(\theta)=\frac{a-1}{a} \sum_{k=0}^{+\infty} a^{-k}\left(C_{\epsilon, 2 L}^{-k} \theta^{k}\right)_{0}
$$

involves inverse powers $C_{\epsilon, 2 L}^{-k}$ of the coupling operator whose entries $\ell_{n, 2 L}^{(k)}$ (see equation (3)) have properties listed in Lemma A.1. In particular, the sign property implies that $\left(C_{\epsilon, 2 L}^{-k} \theta^{k}\right)_{0}(k \geqslant 1)$ is maximum for $\theta^{k}=(10)^{L}$ (i.e. $\theta_{s}^{k}=H\left((-1)^{s}\right)$ for all $\left.s \in \mathbb{Z}_{2 L}\right)$. Moreover the normalization $\sum_{n \in \mathbb{Z}_{2 L}} \ell_{n, 2 L}^{(k)}=$ 1 (Claim A.2) indicates that, among the two homogeneous configurations, $\left(C_{\epsilon, 2 L}^{-k} \theta^{k}\right)_{0}$ reaches its maximum for $\theta^{k}=1^{2 L}$.

Therefore, given any sequence $\theta \in \Omega_{\nu, 2 L}$ such that $\theta_{0}^{0}=0$, the sequence $\tilde{\theta}$ defined by

$$
\tilde{\theta}^{t}=\left\{\begin{array}{cl}
\theta^{0} & \text { if } t=0 \\
(10)^{L} & \text { if } t \geqslant 1 \text { and } \theta^{t} \text { is heterogeneous } \\
1^{2 L} & \text { if } t \geqslant 1 \text { and } \theta^{t} \text { is homogeneous }
\end{array}\right.
$$

also belongs to $\Omega_{\nu, 2 L}$ (because $\sigma\left(\tilde{\theta}^{t}\right)=\sigma\left(\theta^{t}\right)$ for all $t \geqslant 0$ ) and satisfies $\chi_{\epsilon, 2 L}(\theta) \leqslant \chi_{\epsilon, 2 L}(\tilde{\theta})$. Accordingly, the supremum in (8) is attained in the subset where all sequences are composed (for $t \geqslant 1$ ) of configurations that are either $(10)^{L}$ or $1^{2 L}$ and in particular 2-periodic, viz. we have

$$
\begin{aligned}
\sup _{\theta \in \Omega_{\nu, 2 L}: \theta_{0}^{0}=0} \chi_{\epsilon, 2 L}(\theta) & =\sup _{\theta \in \Omega_{\nu, 2 L}: \theta_{0}^{0}=0 \text { and } \theta^{t} \in\left\{(10)^{L}, 1^{2 L}\right\}, \forall t \geqslant 1} \chi_{\epsilon, 2 L}(\theta) \\
& =\sup _{\theta \in \Omega_{\nu, 2}: \theta_{0}^{0}=0 \text { and } \theta^{t} \in\{10,11\}, \forall t \geqslant 1} \chi_{\epsilon, 2}(\theta)
\end{aligned}
$$

Finally, we proved in [7] the existence of a map $\epsilon \mapsto \nu_{\epsilon}$ such that

$$
\sup _{\theta \in \Omega_{\nu, 2}: \theta_{0}^{0}=0 \text { and } \theta^{t} \in\{10,11\}, \forall t \geqslant 1} \chi_{\epsilon, 2}(\theta)<1 / 2 \text { iff } \nu<\nu_{\epsilon}
$$

We also showed that this map is a decreasing Devil's staircase with limit $\lim _{\epsilon \rightarrow \epsilon_{c}^{+}} \nu_{\epsilon}=1$. This completes the proof of the Lemma.

We now study properties of the subshift entropy $h_{\mathrm{top}}\left(\Omega_{\nu, 2 L}, R_{\mathrm{ti}}\right)$. This quantity is an increasing function of $\nu$ that can be characterized by an algebraic equation (see equation (14) in [7] for the equation of $h_{\mathrm{top}}\left(\Omega_{\nu, 2}, R_{\mathrm{ti}}\right)$ ). 
For our purpose here, it is enough (and simpler) to consider the entropy $h_{n, 2 L}:=h_{\mathrm{top}}\left(\Omega_{\frac{n}{n+1}, 2 L}, R_{\mathrm{ti}}\right)$ of the sets $\Omega_{\frac{n}{n+1}, 2 L}$ that consist of all symbolic sequences for which the length of any heterogeneous word is at most $n .^{3}$

Lemma 4.2. For every $n \geqslant 1$, there exists $\delta_{n}>0$ with limit $\lim _{n \rightarrow \infty} \delta_{n}=\log 2$ such that we have $h_{n, 2 L} \geqslant(2 L) \delta_{n}$ for all $L \geqslant 1$.

Proof. $h_{n, 2 L}$ is the exponential rate of increase of the number $N_{t}$ of admissible words of length $t$ in $\Omega_{\frac{n}{n+1}, 2 L}$ [17, 21]. Words in this set conclude with either an homogeneous configuration or an heterogeneous word of length at most $n$.

Given $t \geqslant 1$ (not smaller than $n$ ), let $N_{t}^{0}$ be the number of admissible words of length $t$ with homogeneous suffix and for $k=1, \cdots, n$, let $N_{t}^{1^{k}}$ be the number of admissible words of length $t$ with heterogeneous suffix of length (exactly) $k$. Obviously we have $N_{t}=N_{t}^{0}+\sum_{k=1}^{n} N_{t}^{1^{k}}$ and in order to obtain the growth rate of $N_{t}$, we are going to establish an induction relation for $N_{t}^{0}$.

Firstly, any homogeneous configuration can follow any admissible word to form another admissible word. Since there are two homogeneous configurations, this implies that $N_{t+1}^{0}=2 N_{t}$. Moreover, it is easy to see that we have $N_{t+1}^{1^{k+1}}=\alpha_{2 L} N_{t}^{1^{k}}$ for $k=1, \cdots, n-1$ where $\alpha_{2 L}=2^{2 L}-2$ is the number of heterogeneous configurations. Similarly, we have $N_{t+1}^{1^{1}}=\alpha_{2 L} N_{t}^{0}$. Based on these relations, we get the desired induction

$$
N_{t+1}^{0}=2\left(N_{t}^{0}+\sum_{k=1}^{n} N_{t}^{1^{k}}\right)=2 \sum_{k=0}^{n} \alpha_{2 L}^{k} N_{t-k}^{0}
$$

Since all coefficients in the right hand side are positive, the companion matrix associated with this induction is non-negative and irreducible. By the PerronFrobenius Theorem, it results the quantity $N_{t}^{0}$ has the following behavior

$$
\lim _{t \rightarrow+\infty} \frac{N_{t+1}^{0}}{N_{t}^{0}}=\lambda_{n, 2 L}
$$

where $\lambda_{n, 2 L}$ is the largest positive solution of the equation

$$
\lambda^{n+1}-2 \sum_{k=0}^{n} \alpha_{2 L}^{k} \lambda^{n-k}=0
$$

(the only one for which all components of the corresponding eigenvector are positive). The previous limit implies that

$$
h_{n, 2 L}:=\lim _{t \rightarrow+\infty} \log \left(N_{t}\right)^{1 / t}=\lim _{t \rightarrow+\infty} \log \left(N_{t}^{0}\right)^{1 / t}=\log \lambda_{n, 2 L} .
$$

In order to complete the proof, we use the equation above to show that for every $0<\delta<\log 2$, there exists $n_{\delta} \geqslant 1$ such that for all $n \geqslant n_{\delta}$, we have

$$
h_{n, 2 L} \geqslant(2 L) \delta \forall L \geqslant 1 \text {. }
$$

\footnotetext{
${ }^{3}$ That is to say if $\theta^{t}, \cdots, \theta^{t+k-1}$ are all heterogeneous, then we must have $k \leqslant n$.
} 
We begin to prove that $\lim _{n \rightarrow+\infty} \lambda_{n, 2 L}=2^{2 L}$ for every $L \geqslant 1$. The equation (9) is equivalent to $p_{n, 2 L}(\lambda)=1$ where the function $p_{n, 2 L}$ is defined by

$$
p_{n, 2 L}(\lambda)=2 \sum_{k=0}^{n} \alpha_{2 L}^{k} \lambda^{-(k+1)} .
$$

This function has the following properties

- $\lambda \mapsto p_{n, 2 L}(\lambda)$ is strictly decreasing,

- $p_{n, 2 L}(1)>1$,

- $p_{n+1,2 L}(\lambda)>p_{n, 2 L}(\lambda)$

- $p_{n, 2 L}\left(\alpha_{2 L}+2\right)<p_{\infty, 2 L}\left(\alpha_{2 L}+2\right)=1$

which immediately imply that every sequence $\left\{\lambda_{n, 2 L}\right\}_{n \geqslant 1}$ is strictly increasing in $\left[1, \alpha_{2 L}+2\right]$. Moreover, a direct computation shows that $p_{n, 2 L}\left(\alpha_{2 L}\right)=$ $2 \frac{n+1}{\alpha_{2 L}}$ which yields $\lambda_{2^{2 L-1}-2,2 L}=\alpha_{2 L}$. Accordingly, the series $p_{\infty, 2 L}(\lambda)$ is uniformly convergent for $\lambda_{2^{2 L-1}-1,2 L} \leqslant \lambda \leqslant \alpha_{2 L}+2$. This function is strictly increasing and it easily follows that $\lim _{n \rightarrow+\infty} \lambda_{n, 2 L}=\alpha_{2 L}+2=2^{2 L}$ as desired.

Uniform control on the asymptotic behavior of $\lambda_{n, 2 L}$ will be granted by the following relation which is a consequence of (9)

$$
\lambda_{n, 2 L}^{n+1}=2 \sum_{k=0}^{n} \alpha_{2 L}^{k} \lambda_{n, 2 L}^{n-k}>\alpha_{2 L}^{n}
$$

Accordingly, we have

$$
h_{n, 2 L}>\frac{n}{n+1} \log \alpha_{2 L}>\frac{n}{n+1} \log \left(2^{2 L-1}\right)>(2 L)\left(1-\frac{1}{n+1}-\frac{1}{2 L}\right) \log 2
$$

Now, given $0<\delta<\log 2$, let $L_{\delta}>1$ be such that $\left(1-\frac{1}{L_{\delta}}\right) \log 2 \geqslant \delta$. Using that $\lim _{n \rightarrow+\infty} \lambda_{n, 2 L}=2^{2 L}$, let $\tilde{n}_{\delta}$ be sufficiently large such that

$$
h_{\tilde{n}_{\delta}, 2 L} \geqslant(2 L) \delta, \forall L \in\left\{1, \cdots, 2 L_{\delta}-1\right\} .
$$

The inequality above and the definition of $L_{\delta}$ imply that

$$
h_{2 L_{\delta}-1,2 L}>(2 L) \delta, \forall L \geqslant 2 L_{\delta} .
$$

Letting $n_{\delta}:=\max \left\{\tilde{n}_{\delta}, 2 L_{\delta}-1\right\}$, the monotonicity of the sequences $\left\{h_{n, 2 L}\right\}_{n \geqslant 1}$ finally imply that for every $n \geqslant n_{\delta}$, we have $h_{n, 2 L} \geqslant(2 L) \delta$ for all $L \geqslant 1$.

Proof of Proposition 2.3. This is a direct consequence of the statements in this section. By Lemma 4.1, for every $n \geqslant 1$, there exists $\epsilon_{n}>\epsilon_{c}$ such that all the $\Omega_{\frac{n}{n+1}, 2 L}(L \geqslant 1)$ are admissible for every $\epsilon<\epsilon_{n}$. It results from compactness of these subshifts, from the previous Lemma and from the left inequality in (5), that $h_{\epsilon, 2 L} \geqslant(2 L) \delta_{n}$ for all $L$ when $\epsilon<\epsilon_{n}$ and the Proposition follows from the $\operatorname{limit} \lim _{n \rightarrow \infty} \delta_{n}=\log 2$. In addition, the inequality $\epsilon_{n}>\epsilon_{c}$ for all $n \geqslant 1$ implies that for $\epsilon=\epsilon_{c}$ we have

$$
h_{\epsilon_{c}, 2 L} \geqslant(2 L) \sup _{n \geqslant 1} \delta_{n}=(2 L) \log 2
$$


as claimed in Proposition 2.1.

\section{Coupled map lattices with piecewise increasing individual maps}

In this section, the results obtained for piecewise affine CML are extended by continuation to CML $F_{g, \epsilon, 2 L}$ with piecewise increasing individual map $g$, see equation (2) in Section 2. The strategy consists in showing that the estimates on admissible sequences persist for CML based on small $C^{1}$ perturbations of $f$.

To that goal, we preliminary assert the existence of a symbolic dynamics for piecewise expanding CML $F_{g, \epsilon, 2 L}$. Recall the quantity $a_{g}$ defined in equation (1). Given any real map $g$ as defined before Theorem 2.4 and with $a_{g}>1$, let $\epsilon_{g}:=\frac{a_{g}-1}{2 a_{g}}<1 / 2$. Similarly to as in the piecewise linear case, the code $\theta$ associated with any point $x \in \mathbb{R}^{\mathbb{Z}_{2 L}}$ is the symbolic sequence defined by

$$
\theta_{s}^{t}=H\left(\left(F_{g, \epsilon, 2 L}^{t} x\right)_{s}-1 / 2\right), \forall s \in \mathbb{Z}_{2 L}, t \geqslant 0 .
$$

As before, the existence of the symbolic dynamics is due to the fact that every point in the repeller $\mathcal{I}_{g, \epsilon, 2 L}$ of $F_{g, \epsilon, 2 L}$ is entirely determined by its code, which has to be an admissible sequence.

Lemma 5.1. For every $\epsilon<\epsilon_{g}$ and $L \geqslant 1$, there exists a function $\chi_{g, \epsilon, 2 L}$ : $\Omega_{2 L} \rightarrow \mathbb{R}$ such that $x \in \mathcal{I}_{g, \epsilon, 2 L}$ iff its coordinates are given by

$$
x_{s}=\chi_{g, \epsilon, 2 L} \circ R_{s p}^{s}(\theta), \forall s \in \mathbb{Z}_{2 L}
$$

where the code $\theta$ solves the admissibility equation

$$
\theta_{s}^{t}=H\left(\chi_{g, \epsilon, 2 L} \circ R_{\mathrm{sp}}^{s} \circ R_{\mathrm{ti}}^{t}(\theta)-1 / 2\right), \forall s \in \mathbb{Z}_{2 L}, t \geqslant 0 .
$$

Proof. Following a standard argument, the function $\chi_{g, \epsilon, 2 L}$ is constructed by using the iterated function system associated with the pre-images of $F_{g, \epsilon, 2 L}$. Let $g_{0}$ be the linear extension of the left branch of $g$ to the whole $\mathbb{R}$, i.e.

$$
g_{0}(\mathrm{x})=\left\{\begin{array}{cc}
g(\mathrm{x}) & \text { if } \quad \mathrm{x}<1 / 2 \\
a_{g}(\mathrm{x}-1 / 2)+g(1 / 2-0) & \text { if } \quad \mathrm{x} \geqslant 1 / 2
\end{array}\right.
$$

and similarly, let $g_{1}$ linear extension of the right branch of $g$. The maps $g_{0}$ and $g_{1}$ are invertible and when $\epsilon<\epsilon_{g}$, the coupling operator $C_{\epsilon, 2 L}$ is also invertible. Hence, given any symbolic configuration $\left\{\theta_{s}\right\}:=\left\{\theta_{s}\right\}_{s \in \mathbb{Z}_{2 L}}$, the map $F_{\left\{\theta_{s}\right\}, g, \epsilon, 2 L}$ defined in $\mathbb{R}^{\mathbb{Z}_{2 L}}$ by

$$
\left(F_{\left\{\theta_{s}\right\}, g, \epsilon, 2 L} x\right)_{s}=(1-\epsilon) g_{\theta_{s}}\left(x_{s}\right)+\frac{\epsilon}{2}\left(g_{\theta_{s-1}}\left(x_{s-1}\right)+g_{\theta_{s+1}}\left(x_{s+1}\right)\right), \forall s \in \mathbb{Z}_{2 L}
$$

is invertible and its inverse $F_{\left\{\theta_{s}\right\}, g, \epsilon, 2 L}^{-1}$ given by

$$
\left(F_{\left\{\theta_{s}\right\}, g, \epsilon, 2 L}^{-1} x\right)_{s}=g_{\theta_{s}}^{-1}\left(\left(C_{\epsilon, 2 L}^{-1} x\right)_{s}\right), \forall s \in \mathbb{Z}_{2 L},
$$


is well-defined in $\mathbb{R}^{\mathbb{Z}_{2 L}}$. We claim that the desired function $\chi_{g, \epsilon, 2 L}$ is given by

$$
\chi_{g, \epsilon, 2 L}(\theta)=\lim _{t \rightarrow+\infty}\left(F_{\theta^{0}, g, \epsilon, 2 L}^{-1} \circ F_{\theta^{1}, g, \epsilon, 2 L}^{-1} \circ \cdots \circ F_{\theta^{t}, g, \epsilon, 2 L}^{-1} x\right)_{0} .
$$

We first need to show that the limit exists and does not depend on $x$. Monotonicity and the definition of $a_{g}$ imply the existence of a constant $c_{g}>0$ such that

$$
\left|g_{i}^{-1}(\mathrm{x})\right| \leqslant a_{g}^{-1}|\mathrm{x}|+c_{g}, \forall \mathrm{x} \in \mathbb{R}, i=0,1
$$

from where we get the following bound

$$
\left\|F_{\left\{\theta_{s}\right\}, g, \epsilon, 2 L}^{-1} x\right\| \leqslant\left(a_{g}(1-2 \epsilon)\right)^{-1}\|x\|+c_{g}, \forall x \in \mathbb{R}^{\mathbb{Z}_{2 L}},\left\{\theta_{s}\right\} \in\{0,1\}^{\mathbb{Z}_{2 L}} .
$$

It follows that any sequence $\left\{F_{\theta^{0}, g, \epsilon, 2 L}^{-1} \circ F_{\theta^{1}, g, \epsilon, 2 L}^{-1} \circ \cdots \circ F_{\theta^{t}, g, \epsilon, 2 L}^{-1} x\right\}_{t \geqslant 0}$ is bounded in $\mathbb{R}^{\mathbb{Z}_{2 L}}$. In addition, the definition of $a_{g}$ and $\left\|C_{\epsilon, 2 L}^{-1}\right\|=(1-2 \epsilon)^{-1}$ yield the following inequality

$$
\left\|F_{\left\{\theta_{s}\right\}, g, \epsilon, 2 L}^{-1} x-F_{\left\{\theta_{s}\right\}, g, \epsilon, 2 L}^{-1} y\right\| \leqslant\left(a_{g}(1-2 \epsilon)\right)^{-1}\|x-y\|
$$

for all $x, y \in \mathbb{R}^{\mathbb{Z}_{2 L}}$ and $\left\{\theta_{s}\right\} \in\{0,1\}^{\mathbb{Z}_{2 L}}$. Since $\left(a_{g}(1-2 \epsilon)\right)^{-1}<1$ when $\epsilon<\epsilon_{g}$, one easily deduces that every $\left\{F_{\theta^{0}, g, \epsilon, 2 L}^{-1} \circ F_{\theta^{1}, g, \epsilon, 2 L}^{-1} \circ \cdots \circ F_{\theta^{t}, g, \epsilon, 2 L}^{-1} x\right\}_{t \geqslant 0}$ is also a Cauchy sequence. Therefore it has a limit in $\mathbb{R}^{\mathbb{Z}_{2 L}}$ and by the previous inequality again, this limit does not depend on $x$.

Now, continuity of the maps $g_{0}$ and $g_{1}$ implies

$$
\begin{array}{r}
F_{\theta^{0}, g, \epsilon, 2 L}\left(\lim _{t \rightarrow+\infty} F_{\theta^{0}, g, \epsilon, 2 L}^{-1} \circ F_{\theta^{1}, g, \epsilon, 2 L}^{-1} \circ \cdots \circ F_{\theta^{t}, g, \epsilon, 2 L}^{-1} x\right) \\
=\lim _{t \rightarrow+\infty} F_{\theta^{1}, g, \epsilon, 2 L}^{-1} \circ \cdots \circ F_{\theta^{t}, g, \epsilon, 2 L}^{-1} x .
\end{array}
$$

Since $F_{g, \epsilon, 2 L} x=F_{\left\{\theta_{s}\right\}, g, \epsilon, 2 L} x$ when $x$ satisfies $\theta_{s}=H\left(x_{s}-1 / 2\right)$ for all $s \in$ $\mathbb{Z}_{2 L}$, we conclude that the configuration $\left\{R_{\mathrm{sp}}^{s} \circ \chi_{g, \epsilon, 2 L}(\theta)\right\}_{s \in \mathbb{Z}_{2 L}}=\left\{\chi_{g, \epsilon, 2 L} \circ\right.$ $\left.R_{\mathrm{sp}}^{s}(\theta)\right\}_{s \in \mathbb{Z}_{2 L}}$ belongs to $\mathcal{I}_{g, \epsilon, 2 L}$ when $\theta$ is admissible. Finally, proving that every configuration in the repeller writes $\left\{\chi_{g, \epsilon, 2 L} \circ R_{\mathrm{sp}}^{s}(\theta)\right\}_{s \in \mathbb{Z}_{2 L}}$ is easy and is left to the reader.

With the existence of symbolic dynamics given, our second ingredient is the continuity of the component map $g \mapsto \chi_{g, \epsilon, 2 L}(\theta)$, uniformly in the set of symbolic sequences.

Lemma 5.2. Let $g$ and $\tilde{g}$ be two individual maps with $a_{g}, a_{\tilde{g}}>1$ and such that $\|g-\tilde{g}\| \leqslant \eta$. Then, for every $\epsilon<\min \left\{\epsilon_{g}, \epsilon_{\tilde{g}}\right\}, L \geqslant 1$ and $\theta \in \Omega_{2 L}$, we have

$$
\left|\chi_{g, \epsilon, 2 L}(\theta)-\chi_{\tilde{g}, \epsilon, 2 L}(\theta)\right| \leqslant \frac{\eta}{\bar{a}-(1-2 \epsilon)^{-1}},
$$

where $\bar{a}=\max \left\{a_{g}, a_{\tilde{g}}\right\}$.

Proof: As in the previous proof, consider the linear extensions $g_{0}, g_{1}, \tilde{g}_{0}$ and $\tilde{g}_{1}$ over $\mathbb{R}$ of the branches of the maps $g$ and $\tilde{g}$. Using the definition of $a_{g}$ and the property $\|g-\tilde{g}\|<\eta$, one easily shows that

$$
g_{i}\left(x+\eta / a_{g}\right)>\tilde{g}_{i}(x) \text { and } g_{i}\left(x-\eta / a_{g}\right)<\tilde{g}_{i}(x), \forall x \in \mathbb{R}, i=0,1
$$


which implies the following constraint on the inverse maps

$$
\left\|g_{i}^{-1}-\tilde{g}_{i}^{-1}\right\| \leqslant \eta / a_{g} \text { for } i=0,1 .
$$

By exchanging the roles of $g_{i}$ and $\tilde{g}_{i}$, it follows that the inverse CML introduced in the previous proof satisfy the following inequality

$$
\left\|F_{\left\{\theta_{s}\right\}, g, \epsilon, 2 L}^{-1} x-F_{\left\{\theta_{s}\right\}, \tilde{g}, \epsilon, 2 L}^{-1} x\right\| \leqslant \eta / \bar{a}, \forall x \in \mathbb{R}^{\mathbb{Z}_{2 L}} .
$$

Using the inequality (10), we subsequently obtain

$$
\begin{aligned}
& \left\|F_{\theta^{0}, g, \epsilon, 2 L}^{-1} \circ F_{\theta^{1}, g, \epsilon, 2 L}^{-1} x-F_{\theta^{0}, \tilde{g}, \epsilon, 2 L}^{-1} \circ F_{\theta^{1}, \tilde{g}, \epsilon, 2 L}^{-1} x\right\| \\
\leqslant & \left\|F_{\theta^{0}, g, \epsilon, 2 L}^{-1} \circ F_{\theta^{1}, g, \epsilon, 2 L}^{-1} x-F_{\theta^{0}, \tilde{g}, \epsilon, 2 L}^{-1} \circ F_{\theta^{1}, g, \epsilon, 2 L}^{-1} x\right\| \\
+ & \left\|F_{\theta^{0}, \tilde{g}, \epsilon, 2 L}^{-1} \circ F_{\theta^{1}, g, \epsilon, 2 L}^{-1} x-F_{\theta^{0}, \tilde{g}, \epsilon, 2 L}^{-1} \circ F_{\theta^{1}, \tilde{g}, \epsilon, 2 L}^{-1} x\right\| \\
\leqslant & \eta / \bar{a}+\left(a_{\tilde{g}}(1-2 \epsilon)\right)^{-1}\left\|F_{\theta^{1}, g, \epsilon, 2 L}^{-1} x-F_{\theta^{1}, \tilde{g}, \epsilon, 2 L}^{-1} x\right\|
\end{aligned}
$$

From thereon, an induction and a similar reasoning with the roles of $g$ and $\tilde{g}$ being exchanged directly lead to the desired conclusion.

Proof of Theorem 2.4. We prove the lower bound and the upper bound separately. For the lower bound, we first show the following statement: For every $0<\mu<\epsilon_{c}$, there exists $\eta>0$ such that for every CML $F_{g, \epsilon, 2 L}$ with individual map $g$ satisfying $\|g-f\|+\left|a_{g}-a\right|<\eta$, we have for every $\epsilon<\epsilon_{c}-\mu$

$$
h_{\text {top }}\left(\mathcal{I}_{g, \epsilon, 2 L}, F_{g, \epsilon, 2 L}\right)=(2 L) \log 2, \forall L \geqslant 1 .
$$

In order to prove this fact, we begin to notice that the quantity

$$
\chi_{\epsilon, 2}\left(\left[0 \theta_{1}^{0}\right][10]^{\infty}\right)=\sup _{\theta \in \Omega_{2 L}: \theta_{0}^{0}=0 \text { and } \theta^{t} \in\{10,11\}, \forall t \geqslant 1} \chi_{\epsilon, 2}(\theta)
$$

introduced in the proof of Lemma 3.2 above is a strictly increasing function of $\epsilon$ which coincides with $1 / 2$ for $\epsilon=\epsilon_{c}$. Let then

$$
\zeta:=1 / 2-\chi_{\epsilon_{c}-\mu, 2}\left(\left[0 \theta_{1}^{0}\right][10]^{\infty}\right)>0 .
$$

Let now $\eta_{1}>0$ be sufficiently small such that we have

$$
a_{g}\left(1-2\left(\epsilon_{c}-\mu\right)\right)>1
$$

whenever $a_{g}>a-\eta_{1}\left(\eta_{1}\right.$ exists because $\left.a\left(1-2\left(\epsilon_{c}-\mu\right)\right)>2\right)$. Let $\eta=$ $\min \left\{\eta_{1}, \zeta\left(a-\left(1-2\left(\epsilon_{c}-\mu\right)\right)^{-1}\right)\right\}>0$.

Let $g$ be an individual map such that $\|g-f\|+\left|a_{g}-a\right|<\eta$. Notice that the constraint $\|g-f\|<+\infty$ implies that $a_{g} \leqslant a$; hence $\bar{a}=a$ in this case. The condition $\left|a_{g}-a\right|<\eta$ implies $\epsilon_{c}-\mu<\epsilon_{g}$. Lemma 5.1 then guarantees the existence of the symbolic dynamics of $F_{g, \epsilon, 2 L}$ for every $\epsilon<\epsilon_{c}-\mu$. Moreover, the condition $\|g-f\|<\eta$ and Lemma 5.2 imply that

$\left|\chi_{g, \epsilon, 2 L}(\theta)-\chi_{f, \epsilon, 2 L}(\theta)\right| \leqslant \zeta \frac{a-\left(1-2\left(\epsilon_{c}-\mu\right)\right)^{-1}}{\bar{a}-(1-2 \epsilon)^{-1}} \leqslant \zeta, \forall \theta \in \Omega_{2 L}, \epsilon<\epsilon_{c}-\mu$.

The constant $\zeta$ has been chosen such that for all $L \geqslant 1$

$$
\sup _{\theta \in \Omega_{2 L}: \theta_{0}^{0}=0} \chi_{f, \epsilon, 2 L}(\theta)<1 / 2-\zeta, \forall \epsilon<\epsilon_{c}-\mu .
$$


Therefore, we have

$$
\sup _{\theta \in \Omega_{2 L}: \theta_{0}^{0}=0} \chi_{g, \epsilon, 2 L}(\theta)<1 / 2, \forall \epsilon<\epsilon_{c}-\mu,
$$

from where the relation (11) immediately follows.

In order to obtain a lower bound for $\epsilon>\epsilon_{c}-\mu$, we observe that the quantities

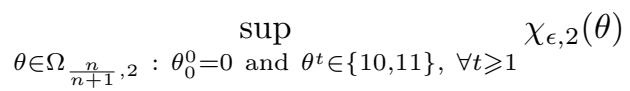

(see end of the proof of Lemma 4.1) are also strictly increasing functions of $\epsilon[7]$. Accordingly, a reasoning similar to the previous one concludes that for every $\mu<\epsilon_{e}$, there exists (another) $\eta>0$ such that for every CML $F_{g, \epsilon, 2 L}$ with individual map $g$ satisfying $\|g-f\|+\left|a_{g}-a\right|<\eta$, we have for every $n \geqslant 0$

$$
h_{\text {top }}\left(\mathcal{I}_{g, \epsilon, 2 L}, F_{g, \epsilon, 2 L}\right) \geqslant h_{\text {top }}\left(\Omega_{\frac{n}{n+1}, 2 L}, R_{\mathrm{ti}}\right) \geqslant(2 L) \delta_{n}, \forall L \geqslant 1, \epsilon<\epsilon_{n}-\mu,
$$

where the $\delta_{n}$ were introduced in Lemma 4.2 and the $\epsilon_{n}$ were defined in the proof of Proposition 2.3 as the maximal coupling strength for which $\Omega_{\frac{n}{n+1}, 2 L} \subset \mathcal{A}_{\epsilon, 2 L}$. The sequence $\left\{\epsilon_{n}\right\}_{n \geqslant 1}$ is strictly decreasing with $\epsilon_{0}=\epsilon_{e}$ and $\lim _{n \rightarrow+\infty} \epsilon_{n}=\epsilon_{c}$. It follows that the lower bound of the Theorem holds with

$$
\underline{\delta}_{\epsilon}:=\left\{\begin{array}{cl}
\log 2 & \text { if } \quad \epsilon<\epsilon_{c}-\mu \\
\delta_{\underline{n}_{\epsilon}} & \text { if } \quad \epsilon>\epsilon_{c}-\mu \text { where } \underline{n}_{\epsilon}:=\max \left\{n \geqslant 1: \epsilon<\epsilon_{n}-\mu\right\}
\end{array}\right.
$$

Moreover, the fact that $\lim _{n \rightarrow+\infty} \epsilon_{n}=\epsilon_{c}$ and $\lim _{n \rightarrow+\infty} \delta_{n}=\log 2$ imply that

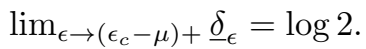

For the upper bound, the reasoning also follows the same lines. We first need an additional restriction on $\mu$ in a way that $\epsilon_{c}+\mu<\epsilon_{e}-\mu$, i.e. we take $\mu<\min \left\{\epsilon_{c},\left(\epsilon_{e}-\epsilon_{c}\right) / 2\right\}$. Then letting now

$$
\zeta:=\chi_{\epsilon_{c}+\mu, 2}\left(\left[0 \theta_{1}^{0}\right][10]^{\infty}\right)-1 / 2>0,
$$

similarly to as above, we set $\eta>0$ (smaller than as before if necessary) such that $\|g-f\|+\left|a_{g}-a\right|<\eta$ implies $\epsilon_{g}>\epsilon_{e}-\mu$ and

$$
\sup _{\theta \in \Omega_{2 L}, L \geqslant 1}\left|\chi_{g, \epsilon, 2 L}(\theta)-\chi_{f, \epsilon, 2 L}(\theta)\right| \leqslant \zeta / 2, \forall \epsilon<\epsilon_{e}-\mu .
$$

As in the proof of Proposition 2.1, this implies that $h_{\mathrm{top}}\left(\mathcal{I}_{g, \epsilon, 2 L}, F_{g, \epsilon, 2 L}\right)<$ $(2 L) \log 2$ for all $L \geqslant 1$ when $\epsilon>\epsilon_{c}+\mu$. Just as in the piecewise linear case, this does not suffice to get extensive decay.

To that goal, the proof of Lemma 3.3 can be repeated mutatis mutandis to show that for every $\epsilon<\epsilon_{e}-\mu$ there exists $\tilde{L}_{\epsilon}, \tilde{T}_{\epsilon} \geqslant 1$ such that any sequence $\theta \in \Omega_{2 L}$ where $\theta_{0}^{0}=0$ and $\theta_{s}^{t}=H\left((-1)^{s}\right)$ for $|s|<L_{\epsilon}$ and $1 \leqslant t \leqslant T_{\epsilon}$ is so that

$$
\left|\chi_{f, \epsilon, 2 L}(\theta)-\chi_{f, \epsilon, 2}\left(\left[0 \theta_{1}^{0}\right][10]^{\infty}\right)\right| \leqslant \zeta / 2
$$

and hence $\chi_{g, \epsilon, 2 L}(\theta)>1 / 2$ which we have show to imply

$$
h_{\text {top }}\left(\mathcal{I}_{g, \epsilon, 2 L}, F_{g, \epsilon, 2 L}\right) \leqslant(2 L)(\log 2-\delta)
$$


provided that $L$ is sufficiently large, say $L \geqslant \tilde{L}$. This concludes the proof of the Theorem.

\section{Appendix A. Properties of the coupling operator $C_{\epsilon, 2 L}$ and its inverse}

The CML mapping $F_{\epsilon, 2 L}$ can be regarded as the composition $C_{\epsilon, 2 L} \circ F_{0,2 L}$ of the coupling operator $C_{\epsilon, 2 L}$ defined in $\mathbb{R}^{\mathbb{Z}_{2 L}}$ by

$$
\left(C_{\epsilon, 2 L} x\right)_{s}=(1-\epsilon) x_{s}+\frac{\epsilon}{2}\left(x_{s-1}+x_{s+1}\right) \forall s \in \mathbb{Z}_{2 L}
$$

and the uncoupled mapping $F_{0,2 L}$ given by

$$
\left(F_{0,2 L} x\right)_{s}=f\left(x_{s}\right) \forall s \in \mathbb{Z}_{2 L} .
$$

\section{A.1. Eigenvalues and the condition for expanding CML}

The coupling operator is linear and commutes with the spatial translations $R_{\mathrm{sp}}$ on the chain $\mathbb{Z}_{2 L}$ defined by $\left(R_{\mathrm{sp}} x\right)_{s}=x_{s-1}$ for all $s \in \mathbb{Z}_{2 L}$. As such, it can be diagonalized in the Fourier basis and a direct calculation shows that its eigenvalues are given by $\hat{c}\left(\epsilon, \frac{k}{2 L}\right)$ for $k \in \mathbb{Z}_{2 L}$ where [2]

$$
\hat{c}(\epsilon, \omega)=1-\epsilon(1-\cos 2 \pi \omega), \forall \omega \in \mathbb{R} / \mathbb{Z} .
$$

(Notice that the eigenvalues for $k \neq 0, L \bmod 2 L$ have multiplicity 2 and the two other ones have simple multiplicity.) In particular, all eigenvalues are nonnegative when $\epsilon \in[0,1 / 2]$ and the smallest eigenvalue is $\hat{c}(\epsilon, 1 / 2)=(1-2 \epsilon)$.

It follows that the eigenvalues of the (constant) derivative of $F_{\epsilon, 2 L}$ outside discontinuities are given by $a \hat{c}\left(\epsilon, \frac{k}{2 L}\right)$. In particular, the smallest eigenvalue is $a \hat{c}(\epsilon, 1 / 2)=a(1-2 \epsilon)$; hence for $\epsilon \in[0,1]$ the CML is expanding iff $a(1-2 \epsilon)>1$ i.e. iff

$$
0 \leqslant \epsilon<\epsilon_{e}:=\frac{a-1}{2 a}
$$

\section{A.2. Coefficients $\ell_{n, 2 L}^{(k)}$ and $\ell_{n}^{(k)}$ and their properties}

The coefficients $\ell_{n, 2 L}^{(k)}$ are the entries of the inverse powers $C_{\epsilon, 2 L}^{-k}$ of the coupling operator, see relation (3) above. The inverse $C_{\epsilon, 2 L}^{-1}$ exists when $\epsilon<1 / 2$ and is a convolution operator [1]. Hence, the coefficients $\ell_{n, 2 L}^{(k)}$ can be regarded as being generated by the following induction

$$
\ell_{n, 2 L}^{(k+1)}=\sum_{m \in \mathbb{Z}_{2 L}} \ell_{m, 2 L}^{(1)} \ell_{n-m, 2 L}^{(k)}, \forall n \in \mathbb{Z}_{2 L}, k \geqslant 0
$$

where $\ell_{n, 2 L}^{(0)}=\delta_{n, 0 \text { mod } 2 L}$ are the entries of the identity in $\mathbb{R}^{\mathbb{Z}_{2 L}}\left(\delta_{n, m}\right.$ being the Kronecker symbol). Together with the same properties for the configuration $\left\{\ell_{n, 2 L}^{(1)}\right\}_{n \in \mathbb{Z}_{2 L}}$, this induction relation implies the following properties. 
Lemma A.1. Every configuration $\left\{\ell_{n, 2 L}^{(k)}\right\}_{n \in \mathbb{Z}_{2 L}}(k \geqslant 1)$ has the properties

$$
\left\{\begin{array}{l}
\ell_{-n, 2 L}^{(k)}=\ell_{n, 2 L}^{(k)} \\
(-1)^{n} \ell_{n, 2 L}^{(k)}>0
\end{array} \quad \forall n \in \mathbb{Z}_{2 L}\right.
$$

and $\left|\ell_{n+1,2 L}^{(k)}\right|<\left|\ell_{n, 2 L}^{(k)}\right|$ for all $n \in\{0, \cdots, L-1\}$.

Proof. We first check that if $\left\{a_{n}\right\}_{n \in \mathbb{Z}_{2 L}}$ and $\left\{b_{n}\right\}_{n \in \mathbb{Z}_{2 L}}$ are two configurations with the properties as in the statement, then the 'convoluted' configuration $\left\{(a * b)_{n}\right\}_{n \in \mathbb{Z}_{2 L}}$ defined by

$$
(a * b)_{n}:=\sum_{m \in \mathbb{Z}_{2 L}} a_{m} b_{n-m}
$$

also satisfies the same properties. Indeed, the equality $(a * b)_{-n}=(a * b)_{n}$ follows from changing $m \mapsto-m$ in the previous sum. Moreover, the property $(-1)^{n}(a * b)_{n}>0$ directly follows from the relation

$$
(-1)^{n}(a * b)_{n}=\sum_{m \in \mathbb{Z}_{2 L}}(-1)^{m} a_{m}(-1)^{n-m} b_{n-m} .
$$

Now, in order to show the inequality $\left|(a * b)_{n+1}\right|<\left|(a * b)_{n},\right|$ for all $n \in$ $\{0, \cdots, L-1\}$, given that the signs of $(a * b)_{n}$ are alternating, it suffices to check that we have

$$
(-1)^{n}\left((a * b)_{n}+(a * b)_{n+1}\right)>0, \forall n \in\{0, \cdots, L-1\} .
$$

To that we goal, we shall need the following inequality

$$
(-1)^{n+m}\left(a_{n-m}+a_{n+m+1}\right)>0, \forall n, m \in\{0, \cdots, L-1\}
$$

that holds for every sequence satisfying the properties of the statement. Indeed, when $n, m \in\{0, \cdots, L-1\}$, we have

$$
-L+1 \leqslant n-m \leqslant L-1 \text { and } 1 \leqslant n+m+1 \leqslant 2 L-1
$$

and we consider separately the cases $n+m+1 \leqslant L$ and $L+1 \leqslant n+m+1$. In the first case, we certainly have $|n-m| \leqslant n+m+1$ and the inequality $\left|a_{n}\right|>\left|a_{n+1}\right|$ for all $n \in\{0, \cdots, L-1\}$ implies

$$
\left|a_{n-m}\right|=\left|a_{|n-m|}\right|>\left|a_{n+m+1}\right| \text {. }
$$

If $L+1 \leqslant n+m+1 \leqslant 2 L-1$, we have $1 \leqslant 2 L-n-m-1 \leqslant L-1$ and $|n-m| \leqslant 2 L-n-m-2$ which implies

$$
\left|a_{n-m}\right|=\left|a_{|n-m|}\right|>\left|a_{2 L-n-m-1}\right|=\left|a_{n+m+1}\right| .
$$

In both cases, it results that

$$
(-1)^{n+m}\left(a_{n-m}+a_{n+m+1}\right)=\left|a_{n-m}\right|-\left|a_{n+m+1}\right|>0 .
$$

as desired. 
Now, using (13) we have

$$
\begin{aligned}
& (a * b)_{n}+(a * b)_{n+1}=\sum_{m \in \mathbb{Z}_{2 L}} a_{n-m}\left(b_{m}+b_{m+1}\right) \\
& =\sum_{m=0}^{L-1} a_{n-m}\left(b_{m}+b_{m+1}\right)+\sum_{m=L}^{2 L-1} a_{n-m}\left(b_{m}+b_{m+1}\right) \\
& =\sum_{m=0}^{L-1}\left(a_{n-m}\left(b_{m}+b_{m+1}\right)+a_{n-2 L+m+1}\left(b_{2 L-m-1}+b_{2 L-m}\right)\right) \\
& =\sum_{m=0}^{L-1}\left(a_{n-m}+a_{n+m+1}\right)\left(b_{m}+b_{m+1}\right) \\
& =(-1)^{n} \sum_{m=0}^{L-1}(-1)^{n+m}\left(a_{n-m}+a_{n+m+1}\right)(-1)^{m}\left(b_{m}+b_{m+1}\right)
\end{aligned}
$$

from where the desired inequality immediately follows.

It remains to show that the original configuration $\left\{\ell_{n, 2 L}^{(1)}\right\}_{n \in \mathbb{Z}_{2 L}}$ possesses the desired properties. To that goal we shall use the following explicit expression of the entries $\ell_{n}^{(1)}$ of the inverse $C_{\epsilon}^{-1}$ of the coupling operator acting in $\ell^{\infty}(\mathbb{Z})$

$$
\ell_{n}^{(1)}=\frac{(-\lambda)^{|n|}}{\gamma}, \forall n \in \mathbb{Z} \text { where } \lambda=\frac{1-\gamma}{1+\gamma} \text { and } \gamma=\sqrt{1-2 \epsilon}<1 .
$$

Now, the expression of $\ell_{n, 2 L}^{(1)}$ reads

$$
\ell_{n, 2 L}^{(1)}=\sum_{m \in \mathbb{Z}} \ell_{n+2 m L}^{(1)}
$$

from which the properties $\ell_{-n, 2 L}^{(1)}=\ell_{n, 2 L}^{(1)}$ and $(-1)^{n} \ell_{n, 2 L}^{(1)}>0$ easily follow. In addition, together with (14), this expression provides an explicit formula for the coefficients, namely

$$
\ell_{n, 2 L}^{(1)}=(-1)^{n} \frac{\lambda^{n}+\lambda^{2 L-n}}{\gamma\left(1-\lambda^{2 L}\right)}, \forall n \in\{0, \cdots, 2 L\}
$$

from which a direct consequence is the inequality $\left|\ell_{n+1,2 L}^{(1)}\right|<\left|\ell_{n, 2 L}^{(1)}\right|$ for all $n \in\{0, \cdots, L-1\}$.

Claim A.2. We have $\sum_{n \in \mathbb{Z}_{2 L}} \ell_{n, 2 L}^{(k)}=1$ for all $k \geqslant 0$.

Proof. From the definition of $C_{\epsilon, 2 L}^{-k}$ we have

$$
\sum_{n \in \mathbb{Z}_{2 L}} \ell_{n, 2 L}^{(k)}=\left(C_{\epsilon, 2 L}^{-k} e_{0}\right)_{m}=1, \forall m \in \mathbb{Z}_{2 L}
$$

where $e_{0}=\{1\}_{s \in \mathbb{Z}_{2 L}}$ is the eigenvector associated with the eigenvalue $\hat{c}(\epsilon, 0)=$ 1. 
Claim A.3. For all $k \geqslant 0$, we have $\sum_{n \in \mathbb{Z}_{2 L}}\left|\ell_{n, 2 L}^{(k)}\right|=(1-2 \epsilon)^{-k}$. Hence, the series $\sum_{k=0}^{+\infty} a^{-k} \sum_{n \in \mathbb{Z}_{2 L}}\left|\ell_{n, 2 L}^{(k)}\right|$ converges for every $\epsilon<\epsilon_{e}$.

Proof. According to Lemma A.1, we have

$$
\sum_{n \in \mathbb{Z}_{2 L}}\left|\ell_{n, 2 L}^{(k)}\right|=\sum_{n \in \mathbb{Z}_{2 L}} \ell_{2 n, 2 L}^{(k)}-\ell_{2 n+1,2 L}^{(k)}=\left(C_{\epsilon, 2 L}^{-k} e_{L}\right)_{0}
$$

where $e_{L}=\left\{(-1)^{s}\right\}_{s \in \mathbb{Z}_{2 L}}$ is the eigenvector associated with the eigenvalue $\hat{c}(\epsilon, 1 / 2)$ (i.e. $k=L$ in the expression $\hat{c}\left(\epsilon, \frac{k}{2 L}\right)$ above). The desired expression then immediately follows from the relation $\hat{c}(\epsilon, 1 / 2)=1-2 \epsilon$. Furthermore, the series $\sum_{k=0}^{+\infty} a^{-k} \sum_{n \in \mathbb{Z}_{2 L}}\left|\ell_{n, 2 L}^{(k)}\right|$ converges when $a(1-2 \epsilon)>1$ i.e. exactly when $\epsilon<\epsilon_{e}$.

Finally the expression of the $\ell_{n, 2}^{(k)}$ is recalled from [9]

$$
\ell_{n, 2}^{(k)}=\frac{1+(-1)^{n}(1-2 \epsilon)^{-k}}{2}, \forall n \in \mathbb{Z}_{2} .
$$

The entries $\ell_{n}^{(k)}$ of the inverse powers $C_{\epsilon}^{-k}$ of the coupling operator acting in $\ell^{\infty}(\mathbb{Z})$ (see proof of Lemma 3.3) have similar properties to the ones in Lemma A.1. In particular, using the definition (14) of the $\ell_{n}^{(1)}$ in an induction based on the convolution relation

$$
\ell_{n}^{(k+1)}=\sum_{m \in \mathbb{Z}} \ell_{m}^{(1)} \ell_{n-m}^{(k)}, \forall n \in \mathbb{Z}, k \geqslant 0
$$

easily yields the properties

$$
\ell_{-n}^{(k)}=\ell_{n}^{(k)},(-1)^{n} \ell_{n}^{(k)}>0 \text { and }\left|\ell_{|n|}^{(k)}\right|>\left|\ell_{|n|+1}^{(k)}\right| \forall n \in \mathbb{Z}, k \geqslant 1 .
$$

Acknowledgment. BF acknowledges stimulating discussions with Lai-Sang Young and is grateful to Courant Institute for hospitality.

\section{References}

[1] V. Afraimovich and B. Fernandez, Topological properties of linearly coupled expanding map lattices, Nonlinearity 13 (2000) 973-993.

[2] J-B. Bardet and B. Fernandez, Extensive escape rate in lattices of weakly coupled expanding maps, Discrete \& Cont. Dynam. Sys. A 31 (2011).

[3] J-B. Bardet and G. Keller, Phase transitions in a piecewise expanding coupled map lattice with linear nearest neighbour coupling, Nonlinearity 19 (2006) 21932210 .

[4] J. Bricmont and A. Kupiainen, Coupled analytic maps, Physica D 8 (1995) 379-396.

[5] L. Bunimovich and Y. Sinai, Space-time chaos in coupled map lattices, Nonlinearity 1 (1988) 491-516. 
[6] J-R. Chazottes and B. Fernandez, ed., Dynamics of Coupled Map Lattices and of Related Spatially Extended Systems, Lec. Notes Phys. 671, Springer-Verlag (2005).

[7] R. Coutinho, B. Fernandez and P. Guiraud, Symbolic dynamics of two coupled lorenz maps: From uncoupled regime to synchronisation, Physica D 237 (2008) $2444-2462$.

[8] B. Fernandez, Global synchronisation in translation invariant coupled map lattices, Int. J. Bif. \& Chaos 18 (2008) 3455-59.

[9] B. Fernandez and P. Guiraud, Route to chaotic synchronisation in coupled map lattices: rigorous results, Discrete \& Cont. Dynam. Sys. B 4 (2004) 435-455.

[10] B. Fernandez and M. Jiang, Coupling two unimodal maps of simple kneading sequences, Ergod. Th. \& Dynam. Sys. 24 (2004) 107-125.

[11] G. Gielis and R. MacKay, Coupled map lattices with phase transitions, Nonlinearity 13 (2000) 867-888.

[12] J. Guckenheimer and P. Holmes, Nonlinear oscillators, dynamical systems and bifurcations of vector fields, Springer-Verlag (1983).

[13] M. Jiang and Y. Pesin, Equilibrium measures for coupled map lattices: Existence, uniqueness and finite-dimensional approximations, Commun. Math. Phys. 193 (1998) 675-711.

[14] J. Jost and M. Joy, Spectral properties and synchronization in coupled map lattices, Phys. Rev. E 65 (2001) 016201.

[15] K. Kaneko, Period-doubling of kink-antikink patterns, quasiperiodicity in antiferro-like structures and spatial intermittency in coupled logistic lattice, Progr. Theor. Phys. 72 (1984) 480-486.

[16] K. Kaneko, ed., Theory and Applications of Coupled Map Lattices, Wiley (1993).

[17] A. Katok and B. Hasselblatt, Introduction to the Modern Theory of Dynamical Systems, Cambridge University Press, Cambridge (1995).

[18] G. Keller, M. Künzle, and T. Nowicki, Some phase transitions in coupled map lattices, Physica D 59 (1992) 39-51.

[19] G. Keller and C. Liverani, Uniqueness of the SRB measure for piecewise expanding weakly coupled map lattices in any dimension, Commun. Math. Phys. 262 (2006) 33-50.

[20] J. Koiller and L-S. Young, Coupled map networks, Nonlinearity 23 (2010) 11211141.

[21] D. Lind and B. Marcus, An Introduction to Symbolic Dynamics and Coding, Cambridge University Press, Cambridge (1995).

[22] P. Lind, J. Corte-Real, and J. Gallas, Modeling velocity in gradient flows with coupled-map lattices with advection, Phys. Rev. E 66 (2002) 016219.

[23] R. MacKay, Dynamics of networks: Features which persist from the uncoupled limit, Stochastic and spatial structures of dynamical systems, Lunel (1996) 81-104.

[24] Y. Oono and S. Puri, Computationally efficient modeling of ordering of quenched phases, Phys. Rev. Lett. 58 (1987) 836-839.

[25] D. Rand, The topological classification of lorenz attractors, Math. Proc. Camb. Phil. Soc. 83 (1978) 451-460. 
[26] C. Robinson, Dynamical Systems, CRC Press (2nd edition), Boca Raton (1999).

[27] R. Solé, J. Valls, and J. Bascompte, Spiral waves, chaos and multiple attractors in lattice models of interacting populations, Phys. Lett. A 166 (1992) 123-128.

[28] I. Waller and R. Kapral, Spatial and temporal structure in systems of coupled nonlinear oscillators, Phys. Rev. A 30 (1984) 2047-2055.

Ricardo Coutinho

Grupo de Física Matemática

Universidade de Lisboa

1649-003 Lisboa, Portugal

and

Departamento de Matemática

Instituto Superior Técnico

Universidade Técnica de Lisboa

1049-001 Lisboa, Portugal

e-mail: Ricardo.Coutinho@math. ist.utl.pt

Bastien Fernandez

Centre de Physique Théorique

CNRS - Aix-Marseille Université

Campus de Luminy

13288 Marseille CEDEX 9, France

e-mail: Bastien.Fernandez@cpt.univ-mrs.fr 